\title{
The resolution of the Navier-Stokes equations in anisotropic spaces
}

\section{Dragos Iftimie}

\begin{abstract}
In this paper we prove global existence and uniqueness for solutions of the 3-dimensional Navier-Stokes equations with small initial data in spaces which are $H^{\delta_{i}}$ in the i-th direction, $\delta_{1}+\delta_{2}+\delta_{3}=$ $1 / 2,-1 / 2<\delta_{i}<1 / 2$ and in a space which is $L^{2}$ in the first two directions and $B_{2,1}^{1 / 2}$ in the third direction, where $H$ and $B$ denote the usual homogeneous Sobolev and Besov spaces.
\end{abstract}

Résumé. Dans cet article on montre l'existence et l'unicité globale des solutions des équations de Navier-Stokes tridimensionnelles pour des données initiales petites dans des espaces qui sont $H^{\delta_{i}}$ dans la ième direction, $\delta_{1}+\delta_{2}+\delta_{3}=1 / 2,-1 / 2<\delta_{i}<1 / 2$ ou dans un espace qui est $L^{2}$ dans les deux premières directions et $B_{2,1}^{1 / 2}$ dans la troisième direction, où $H$ et $B$ sont les espaces de Sobolev et de Besov homogènes habituels.

\section{Introduction.}

In this paper we study the problem of global existence and uniqueness for solutions of the 3-dimensional Navier-Stokes equations. These 
equations are the following

$$
\left\{\begin{array}{l}
\partial_{t} U+U \cdot \nabla U-\nu \Delta U=-\nabla P \\
\operatorname{div} U(t, \cdot)=0, \quad \text { for all } t \geq 0 \\
\left.U\right|_{t=0}=U_{0} .
\end{array}\right.
$$

Here, $U(t, x)$ is a time-dependent three-dimensional vector-field.

The goal of this work is to solve these equations in the spaces

$$
\mathcal{H}^{\delta_{1}, \delta_{2}, \delta_{3}}, \quad \delta_{1}+\delta_{2}+\delta_{3}=\frac{1}{2}, \quad-\frac{1}{2}<\delta_{i}<\frac{1}{2},
$$

and in the space

$$
H B^{0,0,1 / 2},
$$

where the first space is $H^{\delta_{i}}$ in the $\mathrm{i}$-th direction and the second space is $L^{2}$ in the first two directions and $B_{2,1}^{1 / 2}$ in the third direction, where $H^{s}$, respectively $B_{p, q}^{s}$, denote the usual homogeneous Sobolev, respectively Besov, spaces. We are using homogeneous spaces because they are more easy to handle in the case of the Navier-Stokes equations and, in addition, they are larger than the classical ones, so we obtain more general results.

By solving (N-S) in the space $X$ we mean proving the global existence and uniqueness of solutions for small initial data in $X$ and the local existence and uniqueness of solutions for arbitrary initial data in $X$.

The first paragraph is devoted to the study of the spaces $\mathcal{H}^{s_{1}, s_{2}, s_{3}}$, essentially the proof of a product theorem in these spaces. A somewhat similar theorem was proved by M. Sablé-Tougeron in [9] for the Hörmander spaces.

The second paragraph contains the resolution of $(\mathrm{N}-\mathrm{S})$ in

$$
\mathcal{H}^{\delta_{1}, \delta_{2}, \delta_{3}}, \quad \delta_{1}+\delta_{2}+\delta_{3}=\frac{1}{2}, \quad-\frac{1}{2}<\delta_{i}<\frac{1}{2} .
$$

The methods used here are inspired from a paper of J.-Y. Chemin and N. Lerner (see [4]). The case when one of the $\delta_{i}$ equals $1 / 2$ is important but it cannot be studied through our results because $H^{1 / 2}(\mathbb{R})$ is not an algebra. This difficulty is partially avoided by replacing $H^{1 / 2}(\mathbb{R})$ with $B_{2,1}^{1 / 2}(\mathbb{R})$ which has the property to cancel this critical case. And this is how we come to solve (N-S) in the space $H B^{0,0,1 / 2}$ during the third 
paragraph. The same method of replacing $H^{s}$ with $B_{2,1}^{s}$ may be used in the resolution of general hyperbolic symmetric systems. These systems can be solved in the space $H^{s}\left(\mathbb{R}^{d}\right), s>d / 2+1$ but the case $s=d / 2+1$ cannot be proved unless we replace $H^{d / 2+1}$ with $B_{2,1}^{d / 2+1}$ (a short proof is given in the Appendix).

Finally, the last paragraph makes a comparison between this article and the results which are known. We shall see there that the space $H B^{0,0,1 / 2}$ is not imbedded in any of the spaces introduced by H. Kozono and M. Yamazaki in [7], $\mathcal{N}_{p, q, \infty}^{-1+3 / p}$, provided that $1 \leq q \leq p<3 q / 2$, $p>3$. We are not able to prove an imbedding or a nonimbedding if $p \geq 3 q / 2$. The space $\mathcal{H}^{\delta_{1}, \delta_{2}, \delta_{3}}$ is also interesting if we remark, for instance, that we allow negative values for $\delta_{i}$.

The results of this article can be easily extended to an arbitrary dimension, here we consider $\mathbb{R}^{3}$ only for sake of simplicity. In fact, if we work in $\mathbb{R}^{d}$, we can solve (N-S) in the spaces

$$
\mathcal{H}^{\delta_{1}, \delta_{2}, \ldots, \delta_{d}}, \quad \delta_{1}+\delta_{2}+\cdots+\delta_{d}=\frac{d}{2}-1, \quad-\frac{1}{2}<\delta_{i}<\frac{1}{2},
$$

and in the space

$$
H B^{0, \ldots, 0,1 / 2},
$$

where the first space is $H^{\delta_{i}}$ in the $\mathrm{i}$-th direction and the second space is $L^{2}$ in the first $n-1$ directions and $B_{2,1}^{1 / 2}$ in the last one. For instance, we can solve the 2D Navier-Stokes equations with small initial data in $H^{\delta,-\delta}, 0<\delta<1 / 2$, that is in a space of functions which are not square-integrable.

\section{Study of the anisotropic spaces and preliminary results.}

We work in $\mathbb{R}^{3}$ and we denote by $\bar{x}=\left(x_{1}, x_{2}, x_{3}\right)$ the variable in $\mathbb{R}^{3}$. If $\bar{q}=\left(q_{1}, q_{2}, q_{3}\right) \in \mathbb{Z}^{3}$ and $\bar{s}=\left(s_{1}, s_{2}, s_{3}\right) \in \mathbb{R}^{3}$ then we define $\bar{q} \cdot \bar{s}=q_{1} s_{1}+q_{2} s_{2}+q_{3} s_{3}$. Also, if $\bar{\lambda}=\left(\lambda_{1}, \lambda_{2}, \lambda_{3}\right)$ then we note $\bar{\lambda}^{\bar{s}}=\left|\lambda_{1}\right|^{s_{1}}\left|\lambda_{2}\right|^{s_{2}}\left|\lambda_{3}\right|^{s_{3}}$.

Let

$$
\begin{aligned}
\mathcal{L}^{\bar{p}} & =\mathcal{L}^{p_{1}, p_{2}, p_{3}} \\
& =\left\{u \text { such that }\|u\|_{\mathcal{L}^{\bar{p}}} \stackrel{\text { def }}{=}\|\|\left\|u\left(x_{1}, x_{2}, x_{3}\right)\right\|_{L_{x_{3}}^{p_{3}}}\left\|_{L_{x_{2}}^{p_{2}}}\right\|_{\left.L_{x_{1}}^{p_{1}}<\infty\right\}}<\right.
\end{aligned}
$$


and $\ell^{\bar{p}}$ be the analogous space for sequences. Also, when $p=q=r$ we shall note $\ell^{p, p, p}=\ell^{p}$ and $\mathcal{L}^{p, p, p}=L^{p}$. If $u$ is a function $u:(0, T) \times$ $\mathbb{R}^{n} \longrightarrow \mathbb{C}$ then we note

$$
\|u\|_{L_{T}^{p}\left(L^{q}\right)} \stackrel{\text { def }}{=}\|\| u(t, x)\left\|_{L^{q}\left(\mathbb{R}^{n}\right)}\right\|_{L^{p}(0, T)} .
$$

The order of integrations is important, as the following remark shows it:

REMARK 1.1. Let $\left(X_{1}, \mu_{1}\right),\left(X_{2}, \mu_{2}\right)$ be two measure spaces, $1 \leq p \leq q$ and $f: X \times Y \longrightarrow \mathbb{R}$. Then

$$
\|\| f\left(\cdot, x_{2}\right)\left\|_{L^{p}\left(X_{1}, \mu_{1}\right)}\right\|_{L^{q}\left(X_{2}, \mu_{2}\right)} \leq\|\| f\left(x_{1}, \cdot\right)\left\|_{L^{q}\left(X_{2}, \mu_{2}\right)}\right\|_{L^{p}\left(X_{1}, \mu_{1}\right)} \cdot
$$

Indeed

$$
\begin{aligned}
\|\| f\left(\cdot, x_{2}\right)\left\|_{L^{p}\left(X_{1}, \mu_{1}\right)}\right\|_{L^{q}\left(X_{2}, \mu_{2}\right)} & =\left(\left\|\int_{X_{1}} f^{p}\left(\cdot, x_{2}\right) d \mu_{1}\right\|_{L^{q / p}\left(X_{2}, \mu_{2}\right)}\right)^{1 / p} \\
& \leq\left(\int_{X_{1}}\left\|f^{p}\left(x_{1}, \cdot\right)\right\|_{L^{q / p}\left(X_{2}, \mu_{2}\right)} d \mu_{1}\right)^{1 / p} \\
& =\|\| f\left(x_{1}, \cdot\right)\left\|_{L^{q}\left(X_{2}, \mu_{2}\right)}\right\|_{L^{p}\left(X_{1}, \mu_{1}\right)} .
\end{aligned}
$$

The Hölder and Young inequalities for the $\mathcal{L}^{\bar{q}}$ spaces take the form

$$
\|f g\|_{\mathcal{L}^{\bar{p}}} \leq\|f\|_{\mathcal{L}^{\bar{q}}}\|g\|_{\mathcal{L}^{\bar{r}}}
$$

where

$$
\frac{1}{p_{i}}=\frac{1}{q_{i}}+\frac{1}{r_{i}}
$$

for all $i \in\{1,2,3\}$, and

$$
\|f * g\|_{\mathcal{L}^{\bar{a}}} \leq\|f\|_{\mathcal{L}^{\bar{b}}}\|g\|_{\mathcal{L}^{\bar{c}}}
$$

where

$$
1+\frac{1}{a_{i}}=\frac{1}{b_{i}}+\frac{1}{c_{i}}
$$

for all $i \in\{1,2,3\}$.

We can prove a variant of the Littlewood-Paley lemma for the $\mathcal{L}^{\bar{q}}$ spaces: 
Lemma 1.1. If

$$
\begin{aligned}
& \operatorname{supp} \hat{u} \subset B\left(0, r \lambda_{1}, r \lambda_{2}, r \lambda_{3}\right) \\
& \stackrel{\text { def }}{=}\left\{\xi \in \mathbb{R}^{3} \text { such that }\left|\xi_{1}\right|<r \lambda_{1},\left|\xi_{2}\right|<r \lambda_{2},\left|\xi_{3}\right|<r \lambda_{3}\right\} \\
& \text { and } a_{1} \leq b_{1}, a_{2} \leq b_{2}, a_{3} \leq b_{3}, \bar{\alpha}=\left(\alpha_{1}, \alpha_{2}, \alpha_{3}\right) \text { is a multi-index, then } \\
& \qquad\left\|\partial^{\alpha} u\right\|_{\mathcal{L}^{\bar{b}}} \leq C \lambda_{1}^{\alpha_{1}+1 / a_{1}-1 / b_{1}} \lambda_{2}^{\alpha_{2}+1 / a_{2}-1 / b_{2}} \lambda_{3}^{\alpha_{3}+1 / a_{3}-1 / b_{3}}\|u\|_{\mathcal{L}^{\bar{a}}} .
\end{aligned}
$$

Proof. Let $\phi \in C_{0}^{\infty}(\mathbb{R}), \phi$ equal to 1 near the ball of center 0 and radius $r, g=\mathcal{F}^{-1}(\phi)$. Then

$$
\hat{u}(\xi)=\phi\left(\frac{\xi_{1}}{\lambda_{1}}\right) \phi\left(\frac{\xi_{2}}{\lambda_{2}}\right) \phi\left(\frac{\xi_{3}}{\lambda_{3}}\right) \hat{u}(\xi),
$$

and thus

$$
u(x)=\lambda_{1} \lambda_{2} \lambda_{3} \int_{\mathbb{R}^{3}} g\left(\lambda_{1} y_{1}\right) g\left(\lambda_{2} y_{2}\right) g\left(\lambda_{3} y_{3}\right) u(x-y) d y .
$$

Differentiating and using Young's inequality ends the proof.

Before introducing our functional spaces let us recall that the homogeneous Besov spaces are defined to be the closure of compactly supported smooth functions under the norm

$$
\|u\|_{B_{p, q}^{s}} \stackrel{\text { def }}{=}\left\|2^{i s}\right\| \Delta_{i} u\left\|_{L^{p}}\right\|_{\ell^{q}} .
$$

The need of taking the closure of compactly supported smooth functions comes from the fact that the quantity above is only a semi-norm since the "norm" of a polynomial vanishes. Another way of defining these homogeneous spaces is to take equivalence classes of distributions modulo polynomials and to remark that we obtain in that way a real norm. For further details on Besov spaces (homogeneous or not) see $[12]$.

Definition 1.1. We denote by $\mathcal{H}^{s_{1}, s_{2}, s_{3}}=\mathcal{H}^{\bar{s}}$ the closure of compactly supported smooth functions under the norm

$$
|u|_{s_{1}, s_{2}, s_{3}} \stackrel{\text { def }}{=}|u|_{\bar{s}} \stackrel{\text { def }}{=}\left\|\bar{\xi}^{\bar{s}} \hat{u}(\bar{\xi})\right\|_{L^{2}} .
$$


The space $\mathcal{H}^{s_{1}, s_{2}, s_{3}}$ is a Banach space of distributions if $s_{1}<1 / 2$, $s_{2}<1 / 2$ and $s_{3}<1 / 2$.

We denote by $\psi$ a dyadical partition of unity in $\mathbb{R}$, that is a smooth function supported in the ring of center 0 , small radius $3 / 4$, large radius $8 / 3$ and such that $\sum_{q \in \mathbb{Z}} \psi\left(2^{-q} \xi\right)=1$ for all $\xi \neq 0$ (see [1], [5]). Define

$$
\begin{gathered}
\Delta_{q}^{i}=\psi\left(2^{-q} D_{i}\right), \\
S_{q}^{i}=\sum_{p \leq q-1} \Delta_{p}^{i}, \\
S_{\bar{q}}=S_{q_{1}, q_{2}, q_{3}}=S_{q_{1}}^{1} S_{q_{2}}^{2} S_{q_{3}}^{3}, \\
\Delta_{\bar{q}}=\Delta_{q_{1}, q_{2}, q_{3}}=\Delta_{q_{1}}^{1} \Delta_{q_{2}}^{2} \Delta_{q_{3}}^{3}, \\
S_{q}=S_{q, q, q}, \\
\Delta_{q}=S_{q+1}-S_{q} .
\end{gathered}
$$

The following lemmas are easy to prove:

Lemma 1.2. If $u \in \mathcal{H}^{\bar{s}}$ then

$$
|u|_{\bar{s}} \sim\left\|2^{\bar{q} \cdot \bar{s}}\right\| \Delta_{\bar{q}} u\left\|_{L^{2}}\right\|_{\ell^{2}} .
$$

Lemma 1.3. If $u_{\bar{p}}$ is a sequence of functions such that

$$
\begin{aligned}
& \operatorname{supp} \hat{u}_{\bar{p}} \\
& \subset\left\{\frac{1}{\gamma} 2^{p_{1}} \leq\left|\xi_{1}\right| \leq \gamma 2^{p_{1}}, \frac{1}{\gamma} 2^{p_{2}} \leq\left|\xi_{2}\right| \leq \gamma 2^{p_{2}}, \frac{1}{\gamma} 2^{p_{3}} \leq\left|\xi_{3}\right| \leq \gamma 2^{p_{3}}\right\}
\end{aligned}
$$

and

$$
\left\|2^{\bar{p} \cdot \bar{s}}\right\| u_{\bar{p}}\left\|_{L^{2}}\right\|_{\ell^{2}}<\infty
$$

then

$$
u=\sum_{\bar{p}} u_{\bar{p}} \in \mathcal{H}^{s_{1}, s_{2}, s_{3}}
$$

and

$$
|u|_{s_{1}, s_{2}, s_{3}} \leq C\left\|2^{\bar{p} \cdot \bar{s}}\right\| u_{\bar{p}}\left\|_{L^{2}}\right\|_{\ell^{2}}
$$


If $s_{1}>0$ it is enough to assume that

$$
\operatorname{supp} \hat{u}_{\bar{p}} \subset\left\{\left|\xi_{1}\right| \leq \gamma 2^{p_{1}}, \frac{1}{\gamma} 2^{p_{2}} \leq\left|\xi_{2}\right| \leq \gamma 2^{p_{2}}, \frac{1}{\gamma} 2^{p_{3}} \leq\left|\xi_{3}\right| \leq \gamma 2^{p_{3}}\right\} .
$$

If $s_{1}>0$ and $s_{2}>0$ it is enough to assume that

$$
\operatorname{supp} \hat{u}_{\bar{p}} \subset\left\{\left|\xi_{1}\right| \leq \gamma 2^{p_{1}},\left|\xi_{2}\right| \leq \gamma 2^{p_{2}}, \frac{1}{\gamma} 2^{p_{3}} \leq\left|\xi_{3}\right| \leq \gamma 2^{p_{3}}\right\}
$$

If $s_{1}>0, s_{2}>0$ and $s_{3}>0$ it is enough to assume that

$$
\operatorname{supp} \hat{u}_{\bar{p}} \subset\left\{\left|\xi_{1}\right| \leq \gamma 2^{p_{1}},\left|\xi_{2}\right| \leq \gamma 2^{p_{2}},\left|\xi_{3}\right| \leq \gamma 2^{p_{3}}\right\}
$$

The next theorem studies the problem of products in the $\mathcal{H}^{s_{1}, s_{2}, s_{3}}$ spaces.

Theorem 1.1. Let $u \in \mathcal{H}^{\bar{s}}, v \in \mathcal{H}^{\bar{t}}$ such that $s_{i}<1 / 2, t_{i}<1 / 2$, $s_{i}+t_{i}>0, i \in\{1,2,3\}$. Then

$$
u v \in \mathcal{H}^{\bar{s}+\bar{t}-(1 / 2,1 / 2,1 / 2)}
$$

and

$$
|u v|_{\bar{s}+\bar{t}-(1 / 2,1 / 2,1 / 2)} \leq C|u|_{\bar{s}}|v|_{\bar{t}}
$$

Proof. We shall give a proof which imitates the argument for the classical Sobolev spaces. This will be done by introducing 3-dimensional paraproduct operators. We recall the definition of Bony's decomposition

$$
u v=T(u, v)+R(u, v)+\widetilde{T}(u, v),
$$

where

$$
\begin{gathered}
T(u, v)=\sum_{q} S_{q-1} u \Delta_{q} v \\
R(u, v)=\sum_{|p-q| \leq 1} \Delta_{p} u \Delta_{q} v \\
\widetilde{T}(u, v)=T(v, u)
\end{gathered}
$$


(see [1], [5]). It is well-known that $T: H^{s}(\mathbb{R}) \times H^{t}(\mathbb{R}) \longrightarrow H^{s+t-1 / 2}(\mathbb{R})$ is well-defined and continous if $s<1 / 2$. The same is true for $R$ if $s+t>0$. Here we use the analogous of this decomposition

$$
u v=\left(T^{1}+R^{1}+\widetilde{T}^{1}\right)\left(T^{2}+R^{2}+\widetilde{T}^{2}\right)\left(T^{3}+R^{3}+\widetilde{T}^{3}\right)(u, v)
$$

understood as the sum of $3^{3}$ terms. The definition of each term is a straightforward extension of the classical paraproduct and remainder. The reader may give the definition of each term; we give, for instance, the one of the term $T^{1} R^{2} \widetilde{T}^{3}(u, v)$

$$
T^{1} R^{2} \widetilde{T}^{3}(u, v)=\sum_{i=-1}^{1} \sum_{\bar{p}} S_{p_{1}-1}^{1} \Delta_{p_{2}}^{2} \Delta_{p_{3}}^{3} u \Delta_{p_{1}}^{1} \Delta_{p_{2}-i}^{2} S_{p_{3}-1}^{3} v .
$$

We shall prove that each of the $3^{3}$ operators we find is continuous

$$
\mathcal{H}^{\bar{s}} \times \mathcal{H}^{\bar{t}} \longrightarrow \mathcal{H}^{\bar{s}+\bar{t}-(1 / 2,1 / 2,1 / 2)},
$$

under weaker hypothesis than those given in the theorem. More precisely, the conditions to assume are given by the composition of the term in the following manner: if the term contains $T^{i}$ then we have to assumme $s_{i}<1 / 2$; if the term contains $R^{i}$ then we have to assume $s_{i}+t_{i}>0$; if the term contains $\widetilde{T}^{i}$ then we have to assume $t_{i}<1 / 2$. For instance if we want the term $T^{1} R^{2} \widetilde{T}^{3}$ to be continous then we have to assume that $s_{1}<1 / 2, s_{2}+t_{2}>0, t_{3}<1 / 2$. This term is the most difficult to handle so we prove the assertion only on it. We have

$$
T^{1} R^{2} \widetilde{T}^{3}(u, v)=\sum_{i=-1}^{1} \sum_{\bar{p}} w_{\bar{p}}^{i}
$$

where

$$
w_{\bar{p}}^{i}=S_{p_{1}-1}^{1} \Delta_{p_{2}}^{2} \Delta_{p_{3}}^{3} u \Delta_{p_{1}}^{1} \Delta_{p_{2}-i}^{2} S_{p_{3}-1}^{3} v
$$

Using several times the anisotropic form of Hölder's inequality, the definition of the operators $S^{1}$ and $S^{3}$ as well as the anisotropic Littlewood- 
Paley Lemma 1.1 one can show that

$$
\begin{aligned}
& \left\|\Delta_{\bar{q}} w_{\bar{p}}^{i}\right\|_{L^{2}} \\
& \leq 2^{q_{2} / 2}\left\|w_{\bar{p}}^{i}\right\|_{\mathcal{L}^{2,1,2}} \\
& \leq 2^{q_{2} / 2}\left\|S_{p_{1}-1}^{1} \Delta_{p_{2}}^{2} \Delta_{p_{3}}^{3} u\right\|_{\mathcal{L}^{\infty, 2,2}}\left\|\Delta_{p_{1}}^{1} \Delta_{p_{2}-i}^{2} S_{p_{3}-1}^{3} v\right\|_{\mathcal{L}^{2,2, \infty}}
\end{aligned}
$$

$$
\begin{aligned}
& \leq 2^{q_{2} / 2} \sum_{\substack{r_{1} \leq p_{1}-2 \\
r_{3} \leq p_{3}-2}}\left\|\Delta_{r_{1}}^{1} \Delta_{p_{2}}^{2} \Delta_{p_{3}}^{3} u\right\|_{\mathcal{L}^{\infty, 2,2}}\left\|\Delta_{p_{1}}^{1} \Delta_{p_{2}-i}^{2} \Delta_{r_{3}}^{3} v\right\|_{\mathcal{L}^{2,2, \infty}} \\
& \leq 2^{q_{2} / 2} \sum_{\substack{r_{1} \leq p_{1}-2 \\
r_{3} \leq p_{3}-2}} 2^{r_{1} / 2+r_{3} / 2}\left\|\Delta_{r_{1}}^{1} \Delta_{p_{2}}^{2} \Delta_{p_{3}}^{3} u\right\|_{L^{2}}\left\|\Delta_{p_{1}}^{1} \Delta_{p_{2}-i}^{2} \Delta_{r_{3}}^{3} v\right\|_{L^{2}} .
\end{aligned}
$$

Let us introduce

$$
a_{\bar{q}}=2^{\bar{q} \cdot \bar{s}}\left\|\Delta_{\bar{q}} u\right\|_{L^{2}}
$$

and

$$
b_{\bar{q}}=2^{\bar{q} \cdot \bar{t}}\left\|\Delta_{\bar{q}} v\right\|_{L^{2}} .
$$

Since $s_{1}<1 / 2$ and $t_{3}<1 / 2$, inequality (1.1) implies

$$
\begin{aligned}
\left\|\Delta_{\bar{q}} w_{\bar{p}}^{i}\right\|_{L^{2}} \leq & C 2^{q_{2} / 2} 2^{p_{1}\left(1 / 2-s_{1}-t_{1}\right)} 2^{-p_{2}\left(s_{2}+t_{2}\right)} 2^{p_{3}\left(1 / 2-s_{3}-t_{3}\right)} \\
& \cdot\left\|a_{\bar{p}}\right\|_{\ell_{p_{1}}^{\infty}}\left\|b_{p_{1}, p_{2}-i, p_{3}}\right\|_{\ell_{p_{3}}^{\infty}},
\end{aligned}
$$

whence

$$
\begin{aligned}
2^{\bar{q} \cdot(\bar{s}+\bar{t}-(1 / 2,1 / 2,1 / 2))}\left\|\Delta_{\bar{q}} w_{\bar{p}}^{i}\right\|_{L^{2}} \\
\leq C 2^{\left(q_{1}-p_{1}\right)\left(s_{1}+t_{1}-1 / 2\right)} 2^{\left(q_{3}-p_{3}\right)\left(s_{3}+t_{3}-1 / 2\right)} \\
\quad \cdot 2^{\left(q_{2}-p_{2}\right)\left(s_{2}+t_{2}\right)}\left\|a_{\bar{p}}\right\|_{\ell_{p_{1}}^{2}}\left\|b_{p_{1}, p_{2}-i, p_{3}}\right\|_{\ell_{p_{3}}^{2}}
\end{aligned}
$$

Since $\left|p_{1}-q_{1}\right| \leq 1, q_{2}<p_{2},\left|p_{3}-q_{3}\right| \leq 1$ we obtain

$$
\begin{aligned}
& 2^{\bar{q} \cdot(\bar{s}+\bar{t}-(1 / 2,1 / 2,1 / 2))}\left\|\Delta_{\bar{q}} T^{1} R^{2} \widetilde{T}^{3}(u, v)\right\|_{L^{2}} \\
& \quad \leq C \sum_{i=-1}^{1} \sum_{\substack{\left|p_{1}-q_{1}\right| \leq 1 \\
\left|p_{3}-q_{3}\right| \leq 1}} \sum_{p_{2}>q_{2}} 2^{\left(q_{2}-p_{2}\right)\left(s_{2}+t_{2}\right)}\left\|a_{\bar{p}}\right\|_{\ell_{p_{1}}^{2}}\left\|b_{p_{1}, p_{2}-i, p_{3}}\right\|_{\ell_{p_{3}}^{2}} .
\end{aligned}
$$


Taking the $\ell_{q_{1}, q_{3}}^{2}$ norm gives

$$
\begin{aligned}
& \left\|2^{\bar{q} \cdot(\bar{s}+\bar{t}-(1 / 2,1 / 2,1 / 2))}\right\| \Delta_{\bar{q}} T^{1} R^{2} \widetilde{T}^{3}(u, v)\left\|_{L^{2}}\right\|_{\ell_{q_{1}, q_{3}}^{2}} \\
& \quad \leq C \sum_{i=-1}^{1} \sum_{p_{2}>q_{2}} 2^{\left(q_{2}-p_{2}\right)\left(s_{2}+t_{2}\right)}\left\|a_{\bar{p}}\right\|_{\ell_{p_{1}, p_{3}}^{2}}\left\|b_{p_{1}, p_{2}-i, p_{3}}\right\|_{\ell_{p_{1}, p_{3}}^{2}} .
\end{aligned}
$$

Taking the $\ell_{q_{2}}^{2}$ norm, applying Young's inequality and using that $s_{2}+$ $t_{2}>0$ yields

$$
\begin{aligned}
\left\|2^{\bar{q} \cdot(\bar{s}+\bar{t}-(1 / 2,1 / 2,1 / 2))}\right\| \Delta_{\bar{q}} T^{1} R^{2} \widetilde{T}^{3}(u, v)\left\|_{L^{2}}\right\|_{\ell^{2}} \\
\leq C \sum_{i=-1}^{1}\|\| a_{\bar{p}}\left\|_{\ell_{p_{1}, p_{3}}^{2}}\right\| b_{p_{1}, p_{2}-i, p_{3}}\left\|_{\ell_{p_{1}, p_{3}}^{2}}\right\|_{\ell_{p_{2}}^{1}} .
\end{aligned}
$$

Finally, Hölder's inequality implies

$$
\left\|2^{\bar{q} \cdot(\bar{s}+\bar{t}-(1 / 2,1 / 2,1 / 2))}\right\| \Delta_{\bar{q}} T^{1} R^{2} \widetilde{T}^{3}(u, v)\left\|_{L^{2}}\right\|_{\ell^{2}} \leq C\left\|a_{\bar{p}}\right\|_{\ell^{2}}\left\|b_{\bar{p}}\right\|_{\ell^{2}},
$$

that is

$$
\left|T^{1} R^{2} \widetilde{T}^{3}(u, v)\right|_{\bar{s}+\bar{t}-(1 / 2,1 / 2,1 / 2)} \leq C|u|_{\bar{s}}|v|_{\bar{t}} .
$$

This completes the proof.

We shall now adjust this study to the case of the spaces $H B^{s_{1}, s_{2}, s_{3}}$ $=H B^{\bar{s}}$ defined as the closure of compactly supported smooth functions under the norm

$$
|u|_{H B^{\bar{s}}} \stackrel{\text { def }}{=}\left\|2^{\bar{q} \cdot \bar{s}}\right\| \Delta_{\bar{q}} u\left\|_{L^{2}}\right\|_{\ell^{2,2,1}} .
$$

REMARK 1.2. In this definition, when we apply the $\ell^{2,2,1}$ norm, we first take the $\ell^{1}$ norm and afterwards the others, but all the work we do is valid for the spaces $H B$ obtained by appling the $\ell^{2,2,1}$ norm in an arbitrary manner. We choosed this order because, according to Remark 1.1 , this space is the largest.

REMARK 1.3. For all real numbers $s_{1}, s_{2}, s_{3}$ the space $H B^{\bar{s}}$ is strictly included into the space $\mathcal{H}^{\bar{s}}$. Moreover, $H B^{\bar{s}}$ is a Banach space of distributions for $s_{1}<1 / 2, s_{2}<1 / 2$ and $s_{3} \leq 1 / 2$. 
The lemmas 1.2 and 1.3 will modify in an obvious way, only the product theorem is relevant for the (N-S) equations.

Theorem 1.2. Let $u \in H B^{\bar{s}}, v \in H B^{\bar{t}}$ such that $s_{i}<1 / 2, t_{i}<1 / 2$, $s_{i}+t_{i}>0, i \in\{1,2\}$ and $s_{3} \leq 1 / 2, t_{3} \leq 1 / 2, s_{3}+t_{3}>0$. Then

$$
u v \in H B^{\bar{s}+\bar{t}-(1 / 2,1 / 2,1 / 2)}
$$

and

$$
|u v|_{H B^{\bar{s}+\bar{t}-(1 / 2,1 / 2,1 / 2)}} \leq C|u|_{H B^{\bar{s}}}|v|_{H B^{\bar{t}}} .
$$

Proof. The proof is almost identical to the preceding one, the modification, which allows us to take into account the case $s_{3}=1 / 2$ or $t_{3}=1 / 2$ is that the classical paraproduct $T: B_{2,1}^{s}(\mathbb{R}) \times B_{2,1}^{t}(\mathbb{R}) \longrightarrow$ $B_{2,1}^{s+t-1 / 2}(\mathbb{R})$ is well-defined and continous if $s \leq 1 / 2$. Hence, we shall prove that each of the $3^{3}$ operators is continous under the same assumptions as above, with the modification that if a paraproduct in the third direction is involved, then we can allow $s_{3}$ or $t_{3}$, depending on the paraproduct, to be equal to $1 / 2$. The only problem in the proof is that at the end we have to commute some norms which give raise to the wrong inequality. We have to restart from inequality (1.1)

$$
\begin{gathered}
\left\|\Delta_{\bar{q}} w_{\bar{p}}^{i}\right\|_{L^{2}} \leq 2^{q_{2} / 2} \sum_{\substack{r_{1} \leq p_{1}-2 \\
r_{3} \leq p_{3}-2}} 2^{r_{1} / 2+r_{3} / 2}\left\|\Delta_{r_{1}}^{1} \Delta_{p_{2}}^{2} \Delta_{p_{3}}^{3} u\right\|_{L^{2}} \\
\cdot\left\|\Delta_{p_{1}}^{1} \Delta_{p_{2}-i}^{2} \Delta_{r_{3}}^{3} v\right\|_{L^{2}} .
\end{gathered}
$$

Recall that

$$
a_{\bar{q}}=2^{\bar{q} \cdot \bar{s}}\left\|\Delta_{\bar{q}} u\right\|_{L^{2}}
$$

and

$$
b_{\bar{q}}=2^{\bar{q} \cdot \bar{t}}\left\|\Delta_{\bar{q}} v\right\|_{L^{2}} .
$$

We use that $\left|p_{1}-q_{1}\right| \leq 1,\left|p_{3}-q_{3}\right| \leq 1$ to rewrite the last inequality as

$$
\begin{gathered}
2^{\bar{q} \cdot(\bar{s}+\bar{t}-(1 / 2,1 / 2,1 / 2))}\left\|\Delta_{\bar{q}} w_{\bar{p}}^{i}\right\|_{L^{2}} \\
\leq C 2^{\left(s_{2}+t_{2}\right)\left(q_{2}-p_{2}\right)} \sum_{\substack{r_{1} \leq p_{1}-2 \\
r_{3} \leq p_{3}-2}} 2^{\left(r_{1}-p_{1}\right)\left(1 / 2-s_{1}\right)+\left(r_{3}-p_{3}\right)\left(1 / 2-t_{3}\right)} \\
\qquad a_{r_{1}, p_{2}, p_{3}} b_{p_{1}, p_{2}-i, r_{3}} .
\end{gathered}
$$


Now we sum on $i, \bar{p}$ and $q_{3}$ to obtain

$$
\begin{aligned}
& \sum_{q_{3}} 2^{\bar{q} \cdot(\bar{s}+\bar{t}-(1 / 2,1 / 2,1 / 2))}\left\|\Delta_{\bar{q}} T^{1} R^{2} \widetilde{T}^{3}(u, v)\right\|_{L^{2}} \\
& \leq C \sum_{i=-1}^{1} \sum_{\substack{\left|p_{1}-q_{1}\right| \leq 1 \\
p_{2}>q_{2}}} 2^{\left(s_{2}+t_{2}\right)\left(q_{2}-p_{2}\right)} \\
& \cdot \sum_{r_{1} \leq p_{1}-2} 2^{\left(r_{1}-p_{1}\right)\left(1 / 2-s_{1}\right)} \\
& \cdot \sum_{p_{3}} \sum_{r_{3} \leq p_{3}-2} 2^{\left(r_{3}-p_{3}\right)\left(1 / 2-t_{3}\right)} \\
& \cdot a_{r_{1}, p_{2}, p_{3}} b_{p_{1}, p_{2}-i, r_{3}} \\
& \leq C \sum_{i=-1}^{1} \sum_{\substack{\left|p_{1}-q_{1}\right| \leq 1 \\
p_{2}>q_{2}}} 2^{\left(s_{2}+t_{2}\right)\left(q_{2}-p_{2}\right)} \sum_{r_{1} \leq p_{1}-2} 2^{\left(r_{1}-p_{1}\right)\left(1 / 2-s_{1}\right)} \\
& \cdot\left\|a_{r_{1}, p_{2}, p_{3}}\right\|_{\ell_{p_{3}}^{1}}\left\|b_{p_{1}, p_{2}-i, r_{3}}\right\|_{\ell_{r_{3}}^{1}} \\
& \leq C \sum_{\substack { i=-1 \\
\begin{subarray}{c}{\left|p_{1}-q_{1}\right| \leq 1 \\
p_{2}>q_{2}{ i = - 1 \\
\begin{subarray} { c } { | p _ { 1 } - q _ { 1 } | \leq 1 \\
p _ { 2 } > q _ { 2 } } }\end{subarray}} 2^{\left(s_{2}+t_{2}\right)\left(q_{2}-p_{2}\right)}\|\| a_{r_{1}, p_{2}, p_{3}}\left\|_{\ell_{p_{3}}^{1}}\right\|_{\ell_{r_{1}}^{2}}\left\|b_{p_{1}, p_{2}-i, r_{3}}\right\|_{\ell_{r_{3}}^{1}} .
\end{aligned}
$$

Since $\left|p_{1}-q_{1}\right| \leq 1$, applying Holdër's inequality gives

$$
\|\| 2^{\bar{q} \cdot(\bar{s}+\bar{t}-(1 / 2,1 / 2,1 / 2))}\left\|\Delta_{\bar{q}} T^{1} R^{2} \widetilde{T}^{3}(u, v)\right\|_{L^{2}}\left\|_{\ell_{q_{3}}^{1}}\right\|_{\ell_{q_{1}}^{2}}
$$

$$
\leq C \sum_{i=-1}^{1} \sum_{p_{2}>q_{2}} 2^{\left(s_{2}+t_{2}\right)\left(q_{2}-p_{2}\right)}\|\| a_{p_{1}, p_{2}, p_{3}}\left\|_{\ell_{p_{3}}^{1}}\right\|_{\ell_{p_{1}}^{2}}\|\| b_{p_{1}, p_{2}-i, p_{3}}\left\|_{\ell_{p_{3}}^{1}}\right\|_{\ell_{p_{1}}^{2}} .
$$

Using that $q_{2}<p_{2}$ and applying Young's inequality yields

$$
\begin{aligned}
& \left\|2^{\bar{q} \cdot(\bar{s}+\bar{t}-(1 / 2,1 / 2,1 / 2))}\right\| \Delta_{\bar{q}} T^{1} R^{2} \widetilde{T}^{3}(u, v)\left\|_{L^{2}}\right\|_{\ell^{2,2,1}} \\
& \quad \leq C \sum_{i=-1}^{1}\|\|\left\|a_{p_{1}, p_{2}, p_{3}}\right\|_{\ell_{p_{3}}^{1}}\left\|_{\ell_{p_{1}}^{2}}\right\|\left\|b_{p_{1}, p_{2}-i, p_{3}}\right\|_{\ell_{p_{3}}^{1}}\left\|_{\ell_{p_{1}}^{2}}\right\|_{\ell_{p_{2}}^{1}} .
\end{aligned}
$$


Finally, we apply Hölder's inequality to obtain

$$
\begin{aligned}
\left\|2^{\bar{q} \cdot(\bar{s}+\bar{t}-(1 / 2,1 / 2,1 / 2))}\right\| \Delta_{\bar{q}} T^{1} R^{2} \widetilde{T}^{3}(u, v)\left\|_{L^{2}}\right\|_{\ell^{2,2,1}} \\
\leq C\left\|a_{p_{1}, p_{2}, p_{3}}\right\|_{\ell^{2,2,1}}\left\|b_{p_{1}, p_{2}, p_{3}}\right\|_{\ell^{2,2,1}},
\end{aligned}
$$

which implies

$$
\left|T^{1} R^{2} \widetilde{T}^{3}(u, v)\right|_{H B^{\bar{s}+\bar{t}-(1 / 2,1 / 2,1 / 2)}} \leq C|u|_{H B^{\bar{s}}}|v|_{H B^{\bar{t}}} .
$$

This completes the proof for $T^{1} R^{2} \widetilde{T}^{3}$.

Since the third variable plays a special role in the definition of the $H B$ spaces, we show how the same estimates can be modified for other terms. We consider first the term $T^{1} R^{2} R^{3}$. We have

$$
T^{1} R^{2} R^{3}(u, v)=\sum_{i, j=-1}^{1} \sum_{\bar{p}} z_{\bar{p}}^{i, j},
$$

where

$$
z_{\bar{p}}^{i, j}=S_{p_{1}-1}^{1} \Delta_{p_{2}}^{2} \Delta_{p_{3}}^{3} u \Delta_{p_{1}}^{1} \Delta_{p_{2}-i}^{2} \Delta_{p_{3}-j}^{3} v .
$$

As above, we deduce the following inequalities

$$
\begin{aligned}
& \left\|\Delta_{\bar{q}} z_{\bar{p}}^{i, j}\right\|_{L^{2}} \\
& \leq 2^{q_{2} / 2+q_{3} / 2}\left\|z_{\bar{p}}^{i, j}\right\|_{\mathcal{L}^{2,1,1}} \\
& \leq 2^{q_{2} / 2+q_{3} / 2}\left\|S_{p_{1}-1}^{1} \Delta_{p_{2}}^{2} \Delta_{p_{3}}^{3} u\right\|_{\mathcal{L}^{\infty, 2,2} \|}\left\|\Delta_{p_{1}}^{1} \Delta_{p_{2}-i}^{2} \Delta_{p_{3}-j}^{3} v\right\|_{\mathcal{L}^{2,2,2}}
\end{aligned}
$$

$$
\begin{aligned}
& \leq 2^{q_{2} / 2+q_{3} / 2} \sum_{r_{1} \leq p_{1}-2}\left\|\Delta_{r_{1}}^{1} \Delta_{p_{2}}^{2} \Delta_{p_{3}}^{3} u\right\|_{\mathcal{L}^{\infty, 2,2}}\left\|\Delta_{p_{1}}^{1} \Delta_{p_{2}-i}^{2} \Delta_{p_{3}-j}^{3} v\right\|_{\mathcal{L}^{2,2,2}} \\
& \leq 2^{q_{2} / 2+q_{3} / 2} \sum_{r_{1} \leq p_{1}-2} 2^{r_{1} / 2}\left\|\Delta_{r_{1}}^{1} \Delta_{p_{2}}^{2} \Delta_{p_{3}}^{3} u\right\|_{L^{2}}\left\|\Delta_{p_{1}}^{1} \Delta_{p_{2}-i}^{2} \Delta_{p_{3}-j}^{3} v\right\|_{L^{2}} .
\end{aligned}
$$

Since $\left|p_{1}-q_{1}\right| \leq 1$, it follows that

$$
\begin{aligned}
2^{\bar{q} \cdot(\bar{s}+\bar{t}-(1 / 2,1 / 2,1 / 2))}\left\|\Delta_{\bar{q}} z_{\bar{p}}^{i, j}\right\|_{L^{2}} & \\
\leq & C 2^{\left(s_{2}+t_{2}\right)\left(q_{2}-p_{2}\right)+\left(s_{3}+t_{3}\right)\left(q_{3}-p_{3}\right)} \\
& \cdot \sum_{r_{1} \leq p_{1}-2} 2^{\left(r_{1}-p_{1}\right)\left(1 / 2-s_{1}\right)} a_{r_{1}, p_{2}, p_{3}} b_{p_{1}, p_{2}-i, p_{3}-j} \cdot
\end{aligned}
$$


Now we sum on $i, j, \bar{p}$ and $q_{3}$ to obtain

$$
\begin{aligned}
& \sum_{q_{3}} 2^{\bar{q} \cdot(\bar{s}+\bar{t}-(1 / 2,1 / 2,1 / 2))}\left\|\Delta_{\bar{q}} T^{1} R^{2} R^{3}(u, v)\right\|_{L^{2}} \\
& \leq C \sum_{\substack { i, j=-1 \\
\begin{subarray}{c}{\left|p_{1}-q_{1}\right| \leq 1 \\
p_{2}>q_{2}{ i , j = - 1 \\
\begin{subarray} { c } { | p _ { 1 } - q _ { 1 } | \leq 1 \\
p _ { 2 } > q _ { 2 } } }\end{subarray}} 2^{\left(s_{2}+t_{2}\right)\left(q_{2}-p_{2}\right)} \\
& \cdot \sum_{r_{1} \leq p_{1}-2} 2^{\left(r_{1}-p_{1}\right)\left(1 / 2-s_{1}\right)} \\
& \cdot \sum_{q_{3}} \sum_{p_{3}>q_{3}} 2^{\left(s_{3}+t_{3}\right)\left(q_{3}-p_{3}\right)} \cdot a_{r_{1}, p_{2}, p_{3}} b_{p_{1}, p_{2}-i, p_{3}-j} .
\end{aligned}
$$

Applying Young's inequality gives

$$
\begin{aligned}
\sum_{q_{3}} \sum_{p_{3}>q_{3}} 2^{\left(s_{3}+t_{3}\right)\left(q_{3}-p_{3}\right)} & a_{r_{1}, p_{2}, p_{3}} b_{p_{1}, p_{2}-i, p_{3}-j} \\
& \leq C\left\|a_{r_{1}, p_{2}, p_{3}} b_{p_{1}, p_{2}-i, p_{3}}\right\|_{\ell_{p_{3}}^{1}} \\
& \leq C\left\|a_{r_{1}, p_{2}, p_{3}}\right\|_{\ell_{p_{3}}^{1}}\left\|b_{p_{1}, p_{2}-i, p_{3}}\right\|_{\ell_{p_{3}}^{1}} .
\end{aligned}
$$

It follows that

$$
\begin{aligned}
& \sum_{q_{3}} 2^{\bar{q} \cdot(\bar{s}+\bar{t}-(1 / 2,1 / 2,1 / 2))}\left\|\Delta_{\bar{q}} T^{1} R^{2} R^{3}(u, v)\right\|_{L^{2}} \\
& \leq C \sum_{\substack { i=-1 \\
\begin{subarray}{c}{p_{1}-q_{1} \mid \leq 1 \\
p_{2}>q_{2}{ i = - 1 \\
\begin{subarray} { c } { p _ { 1 } - q _ { 1 } | \leq 1 \\
p _ { 2 } > q _ { 2 } } }\end{subarray}} 2^{\left(s_{2}+t_{2}\right)\left(q_{2}-p_{2}\right)} \\
& \cdot \sum_{r_{1} \leq p_{1}-2} 2^{\left(r_{1}-p_{1}\right)\left(1 / 2-s_{1}\right)}\left\|a_{r_{1}, p_{2}, p_{3}}\right\|_{\ell_{p_{3}}^{1}}\left\|b_{p_{1}, p_{2}-i, p_{3}}\right\|_{\ell_{p_{3}}^{1}} \\
& \leq C \sum_{i=-1} \sum_{\substack{\left|p_{1}-q_{1}\right| \leq 1 \\
p_{2}>q_{2}}} 2^{\left(s_{2}+t_{2}\right)\left(q_{2}-p_{2}\right)}\|\| a_{r_{1}, p_{2}, p_{3}}\left\|_{\ell_{p_{3}}^{1}}\right\| \ell_{\ell_{1}}^{2}\left\|b_{p_{1}, p_{2}-i, p_{3}}\right\|_{\ell_{p_{3}}^{1}} .
\end{aligned}
$$


Since $\left|p_{1}-q_{1}\right| \leq 1$, applying Holdër's inequality gives

$$
\begin{aligned}
& \|\| 2^{\bar{q} \cdot(\bar{s}+\bar{t}-(1 / 2,1 / 2,1 / 2))}\left\|\Delta_{\bar{q}} T^{1} R^{2} R^{3}(u, v)\right\|_{L^{2}}\left\|_{\ell_{q_{3}}^{1}}\right\|_{\ell_{q_{1}}^{2}} \\
& \leq C \sum_{i=-1}^{1} \sum_{p_{2}>q_{2}} 2^{\left(s_{2}+t_{2}\right)\left(q_{2}-p_{2}\right)} \\
& \qquad\|\| a_{p_{1}, p_{2}, p_{3}}\left\|_{\ell_{p_{3}}^{1}}\right\|_{\ell_{p_{1}}^{2}}\|\| b_{p_{1}, p_{2}-i, p_{3}}\left\|_{\ell_{p_{3}}^{1}}\right\|_{\ell_{p_{1}}^{2}} .
\end{aligned}
$$

This inequality is similar to (1.6), so we can continue likewise to obtain the result on $T^{1} R^{2} R^{3}$.

Finally, we give the proof for the term $T^{1} \widetilde{T}^{2} R^{3}$. As above we have

$$
T^{1} \widetilde{T}^{2} R^{3}(u, v)=\sum_{i=-1}^{1} \sum_{\bar{p}} \alpha_{\bar{p}}^{i},
$$

where

$$
\alpha_{\bar{p}}^{i}=S_{p_{1}-1}^{1} \Delta_{p_{2}}^{2} \Delta_{p_{3}}^{3} u \Delta_{p_{1}}^{1} S_{p_{2}-1}^{2} \Delta_{p_{3}-i}^{3} v .
$$

As above, we deduce the following inequalities

$$
\begin{aligned}
& \left\|\Delta_{\bar{q}} \alpha_{\bar{p}}^{i}\right\|_{L^{2}} \\
& \leq 2^{q_{3} / 2}\left\|\alpha_{\bar{p}}^{i}\right\|_{\mathcal{L}^{2,2,1}} \\
& \leq 2^{q_{3} / 2}\left\|S_{p_{1}-1}^{1} \Delta_{p_{2}}^{2} \Delta_{p_{3}}^{3} u\right\|_{\mathcal{L}^{\infty, 2,2}}\left\|\Delta_{p_{1}}^{1} S_{p_{2}-1}^{2} \Delta_{p_{3}-i}^{3} v\right\|_{\mathcal{L}^{2, \infty, 2}}
\end{aligned}
$$

$$
\begin{aligned}
& \leq 2^{q_{3} / 2} \sum_{\substack{r_{1} \leq p_{1}-2 \\
r_{2} \leq p_{2}-1}}\left\|\Delta_{r_{1}}^{1} \Delta_{p_{2}}^{2} \Delta_{p_{3}}^{3} u\right\|_{\mathcal{L}^{\infty, 2,2}}\left\|\Delta_{p_{1}}^{1} \Delta_{r_{2}}^{2} \Delta_{p_{3}-i}^{3} v\right\|_{\mathcal{L}^{2, \infty, 2}} \\
& \leq 2^{q_{3} / 2} \sum_{\substack{r_{1} \leq p_{1}-2 \\
r_{2} \leq p_{2}-1}} 2^{r_{1} / 2+r_{2} / 2}\left\|\Delta_{r_{1}}^{1} \Delta_{p_{2}}^{2} \Delta_{p_{3}}^{3} u\right\|_{L^{2}}\left\|\Delta_{p_{1}}^{1} \Delta_{r_{2}}^{2} \Delta_{p_{3}-i}^{3} v\right\|_{L^{2}}
\end{aligned}
$$

Since $\left|p_{1}-q_{1}\right| \leq 1$ and $\left|p_{2}-q_{2}\right| \leq 1$ it follows that

$$
\begin{gathered}
2^{\bar{q} \cdot(\bar{s}+\bar{t}-(1 / 2,1 / 2,1 / 2))}\left\|\Delta_{\bar{q}} \alpha_{\bar{p}}^{i}\right\|_{L^{2}} \\
\leq C 2^{\left(s_{3}+t_{3}\right)\left(q_{3}-p_{3}\right)} \sum_{\substack{r_{1} \leq p_{1}-2 \\
r_{3} \leq p_{3}-2}} 2^{\left(r_{1}-p_{1}\right)\left(1 / 2-s_{1}\right)+\left(r_{2}-p_{2}\right)\left(1 / 2-t_{2}\right)} \\
\qquad a_{r_{1}, p_{2}, p_{3}} b_{p_{1}, r_{2}, p_{3}-i} .
\end{gathered}
$$


Now we sum on $i, \bar{p}$ and $q_{3}$ to obtain

$$
\begin{aligned}
& \sum_{q_{3}} 2^{\bar{q} \cdot(\bar{s}+\bar{t}-(1 / 2,1 / 2,1 / 2))}\left\|\Delta_{\bar{q}} T^{1} \widetilde{T}^{2} R^{3}(u, v)\right\|_{L^{2}} \\
& \leq C \sum_{\substack { i=-1 \\
\begin{subarray}{c}{\left|p_{1}-q_{1}\right| \leq 1 \\
\left|p_{2}-q_{2}\right| \leq 1{ i = - 1 \\
\begin{subarray} { c } { | p _ { 1 } - q _ { 1 } | \leq 1 \\
| p _ { 2 } - q _ { 2 } | \leq 1 } }\end{subarray}} \sum_{\substack{r_{1} \leq p_{1}-2 \\
r_{2} \leq p_{2}-2}} 2^{\left(r_{1}-p_{1}\right)\left(1 / 2-s_{1}\right)+\left(r_{2}-p_{2}\right)\left(1 / 2-t_{2}\right)} \\
& \cdot \sum_{q_{3}} \sum_{p_{3}>q_{3}} 2^{\left(s_{3}+t_{3}\right)\left(q_{3}-p_{3}\right)} a_{r_{1}, p_{2}, p_{3}} b_{p_{1}, r_{2}, p_{3}-i} .
\end{aligned}
$$

Applying Young's inequality gives

$$
\begin{aligned}
\sum_{q_{3}} \sum_{p_{3}>q_{3}} 2^{\left(s_{3}+t_{3}\right)\left(q_{3}-p_{3}\right)} & a_{r_{1}, p_{2}, p_{3}} b_{p_{1}, r_{2}, p_{3}-i} \\
& \leq C\left\|a_{r_{1}, p_{2}, p_{3}} b_{p_{1}, r_{2}, p_{3}-i}\right\|_{\ell_{p_{3}}^{1}} \\
& \leq C\left\|a_{r_{1}, p_{2}, p_{3}}\right\|_{\ell_{p_{3}}^{1}}\left\|b_{p_{1}, r_{2}, p_{3}}\right\|_{\ell_{p_{3}}^{1}} .
\end{aligned}
$$

It follows that

$$
\begin{aligned}
& \sum_{q_{3}} 2^{\bar{q} \cdot(\bar{s}+\bar{t}-(1 / 2,1 / 2,1 / 2))}\left\|\Delta_{\bar{q}} T^{1} \widetilde{T}^{2} R^{3}(u, v)\right\|_{L^{2}} \sum_{\substack{\left|p_{1}-q_{1}\right| \leq 1 \\
\left|p_{2}-q_{2}\right| \leq 1}} 2_{\substack{r_{1} \leq p_{1}-2 \\
r_{2} \leq p_{2}-1}}^{\left(r_{1}-p_{1}\right)\left(1 / 2-s_{1}\right)+\left(r_{2}-p_{2}\right)\left(1 / 2-t_{2}\right)} \\
& \leq\left\|a_{r_{1}, p_{2}, p_{3}}\right\|_{\ell_{p_{3}}^{1}}\left\|b_{p_{1}, r_{2}, p_{3}}\right\|_{\ell_{p_{3}}^{1}} \\
& \leq C \sum_{\substack{\left|p_{1}-q_{1}\right| \leq 1 \\
\left|p_{2}-q_{2}\right| \leq 1}}\|\| a_{r_{1}, p_{2}, p_{3}}\left\|_{\ell_{p_{3}}^{1}}\right\|_{\ell_{r_{1}}^{2}}\|\| b_{p_{1}, r_{2}, p_{3}}\left\|_{\ell_{p_{3}}^{1}}\right\|_{\ell_{r_{2}}^{2}} .
\end{aligned}
$$

Using again that $\left|p_{1}-q_{1}\right| \leq 1,\left|p_{2}-q_{2}\right| \leq 1$ and taking the $\ell_{q_{1}, q_{2}}^{2}$ norm yields

$$
\begin{aligned}
\left\|2^{\bar{q} \cdot(\bar{s}+\bar{t}-(1 / 2,1 / 2,1 / 2))}\right\| \Delta_{\bar{q}} T^{1} \widetilde{T}^{2} R^{3}(u, v) \|_{L^{2}} & \|_{\ell^{2,2,1}} \\
& \leq C\left\|a_{\bar{p}}\right\|_{\ell^{2,2,1}}\left\|b_{\bar{p}}\right\|_{\ell^{2,2,1}},
\end{aligned}
$$

that is

$$
\left|T^{1} \widetilde{T}^{2} R^{3}(u, v)\right|_{H B^{\bar{s}+\bar{t}-(1 / 2,1 / 2,1 / 2)}} \leq C|u|_{H B^{\bar{s}}}|v|_{H B^{\bar{t}}}
$$


This completes the proof.

\section{Resolution of (N-S) in the $\mathcal{H}^{s_{1}, s_{2}, s_{3}}$ spaces.}

Let $-1 / 2<\delta_{i}<1 / 2, i \in\{1,2,3\}, \delta_{1}+\delta_{2}+\delta_{3}=1 / 2$. Then there exist nonnegative numbers $a_{1}, a_{2}, a_{3}$ such that

(2.1) $0<\delta_{i}+a_{i}<\frac{1}{2}, \quad$ for all $i \in\{1,2,3\}$ and $a_{1}+a_{2}+a_{3}=\frac{1}{2}$

(one can choose $a_{i}=1 / 4-\delta_{i} / 2$ ). We shall prove the following theorems:

Theorem 2.1 (global existence and uniqueness). There exists $C>0$ such that if $\operatorname{div} u_{0}=0, u_{0} \in \mathcal{H}^{\bar{\delta}}$ and $|u|_{\bar{\delta}}<C \nu$ then the (N-S) equations have a unique solution in

$$
L^{4}(] 0, \infty\left[; \mathcal{H}^{\bar{\delta}+\bar{a}}\right) \cap L^{\infty}(] 0, \infty\left[; \mathcal{H}^{\bar{\delta}}\right)
$$

Moreover, the solution satisfies $u \in \mathcal{C}\left(\left[0, \infty\left[; \mathcal{H}^{\bar{\delta}}\right)\right.\right.$.

Theorem 2.2 (local existence and uniqueness). If $\operatorname{div} u_{0}=0$ and $u_{0} \in \mathcal{H}^{\bar{\delta}}$ then a time $T>0$ and a unique solution of $(\mathrm{N}-\mathrm{S})$ on $[0, T]$ exist so that

$$
u \in L^{4}(] 0, T\left[; \mathcal{H}^{\bar{\delta}+\bar{a}}\right) \cap \mathcal{C}\left(\left[0, T\left[; \mathcal{H}^{\bar{\delta}}\right)\right.\right.
$$

The uniqueness is proved at the end. The global existence is proved in the same time with the local existence. In fact, we shall prove a better result valid for the space $H_{T}$ defined as the closure of compactly supported smooth functions under the norm

$$
\|u\|_{H_{T}} \stackrel{\text { def }}{=}\|\| 2^{\bar{q} \cdot(\bar{\delta}+\bar{a})} \Delta_{\bar{q}} u\left\|_{L_{T}^{4}\left(L^{2}\right)}\right\|_{\ell^{2}} .
$$

Theorem 2.3. Let $\operatorname{div} u_{0}=0$ and $u_{0} \in \mathcal{H}^{\bar{\delta}}$. Then there exist $T>0$ and a solution of $(\mathrm{N}-\mathrm{S})$ on $[0, T]$ which verifies $u \in H_{T}$.

REMARK 2.1. We have $H_{T} \hookrightarrow L^{4}(] 0, T\left[; \mathcal{H}^{\bar{\delta}+\bar{a}}\right)$. 
Indeed, from Remark 1.1 we infer

$$
\begin{aligned}
\|u\|_{L^{4}(] 0, T\left[; \mathcal{H}^{\bar{\delta}+\bar{a})}\right.} & =\|\| 2^{\bar{q} \cdot(\bar{\delta}+\bar{a})}\left\|\Delta_{\bar{q}} u\right\|_{L^{2}}\left\|_{\ell^{2}}\right\|_{L^{4}} \\
& \leq\|\| 2^{\bar{q} \cdot(\bar{\delta}+\bar{a})} \Delta_{\bar{q}} u\left\|_{L_{T}^{4}\left(L^{2}\right)}\right\|_{\ell^{2}} \\
& =\|u\|_{H_{T}} .
\end{aligned}
$$

Proof of Theorem 2.3. We approach $u_{0}$ with the sequence $u_{0}^{n}=$ $S_{n} u_{0}$, where $S_{n}$ is the classical $S_{n}$ in $\mathbb{R}^{3}$. Let $u_{n}$ be the local regular solution of (N-S) with initial data $u_{0}^{n}$ (for the existence of $u_{n}$ see [6], [11]). For each $n$ we apply $\Delta_{\bar{q}}$ at $(\mathrm{N}-\mathrm{S})$ and we multiply by $\Delta_{\bar{q}} u_{n}$ to obtain

$$
\begin{aligned}
\frac{d}{d t}\left\|\Delta_{\bar{q}} u_{n}\right\|_{L^{2}}^{2}+\nu\left\|\nabla \Delta_{\bar{q}} u_{n}\right\|_{L^{2}}^{2} & \leq C\left|\left\langle\Delta_{\bar{q}}\left(u_{n} \nabla u_{n}\right) \mid \Delta_{\bar{q}} u_{n}\right\rangle\right| \\
& =C\left|\left\langle\Delta_{\bar{q}}\left(\operatorname{div}\left(u_{n} \otimes u_{n}\right)\right) \mid \Delta_{\bar{q}} u_{n}\right\rangle\right| .
\end{aligned}
$$

The localization of the Fourier transform of $\Delta_{\bar{q}} u_{n}$ enables us to say that

$$
\begin{aligned}
\left\|\nabla \Delta_{\bar{q}} u_{n}\right\|_{L^{2}}^{2} & =\left\|\partial_{1} \Delta_{\bar{q}} u_{n}\right\|_{L^{2}}^{2}+\left\|\partial_{2} \Delta_{\bar{q}} u_{n}\right\|_{L^{2}}^{2}+\left\|\partial_{3} \Delta_{\bar{q}} u_{n}\right\|_{L^{2}}^{2} \\
& \geq C 4^{q_{1}}\left\|\Delta_{\bar{q}} u_{n}\right\|_{L^{2}}^{2}+C 4^{q_{2}}\left\|\Delta_{\bar{q}} u_{n}\right\|_{L^{2}}^{2}+C 4^{q_{3}}\left\|\Delta_{\bar{q}} u_{n}\right\|_{L^{2}}^{2} \\
& =C\left(4^{q_{1}}+4^{q_{2}}+4^{q_{3}}\right)\left\|\Delta_{\bar{q}} u_{n}\right\|_{L^{2}}^{2} .
\end{aligned}
$$

Moreover, we have from Theorem 1.1 that if $u_{n} \in \mathcal{H}^{\bar{\delta}+\bar{a}}$, then $u_{n} \otimes u_{n} \in$ $\mathcal{H}^{2 \bar{\delta}+2 \bar{a}-(1 / 2,1 / 2,1 / 2)}$. Thus we can write

$$
\operatorname{div}\left(u_{n} \otimes u_{n}\right)=\sum_{j=1}^{3} w_{j}
$$

where

$$
\begin{aligned}
& \left|w_{1}\right|_{2 \bar{\delta}+2 \bar{a}-(3 / 2,1 / 2,1 / 2)} \leq C\left|u_{n} \otimes u_{n}\right|_{2 \bar{\delta}+2 \bar{a}-(1 / 2,1 / 2,1 / 2)} \leq C\left|u_{n}\right|_{\bar{\delta}+\bar{a}}^{2}, \\
& \left|w_{2}\right|_{2 \bar{\delta}+2 \bar{a}-(1 / 2,3 / 2,1 / 2)} \leq C\left|u_{n} \otimes u_{n}\right|_{2 \bar{\delta}+2 \bar{a}-(1 / 2,1 / 2,1 / 2)} \leq C\left|u_{n}\right|_{\bar{\delta}+\bar{a}}^{2}, \\
& \left|w_{3}\right|_{2 \bar{\delta}+2 \bar{a}-(1 / 2,1 / 2,3 / 2)} \leq C\left|u_{n} \otimes u_{n}\right|_{2 \bar{\delta}+2 \bar{a}-(1 / 2,1 / 2,1 / 2)} \leq C\left|u_{n}\right|_{\bar{\delta}+\bar{a}}^{2} .
\end{aligned}
$$


It follows that

$$
\begin{aligned}
\left|\left\langle\Delta_{\bar{q}}\left(u_{n} \nabla u_{n}\right) \mid \Delta_{\bar{q}} u_{n}\right\rangle\right| & \\
\leq C & \left(2^{-\bar{q} \cdot(2 \bar{\delta}+2 \bar{a}-(3 / 2,1 / 2,1 / 2))}+2^{-\bar{q} \cdot(2 \bar{\delta}+2 \bar{a}-(1 / 2,3 / 2,1 / 2))}\right. \\
& \left.+2^{-\bar{q} \cdot(2 \bar{\delta}+2 \bar{a}-(1 / 2,1 / 2,3 / 2))}\right) a_{\bar{q}}\left\|\Delta_{\bar{q}} u_{n}\right\|_{L^{2}}\left|u_{n}\right| \frac{2}{\delta}+\bar{a}
\end{aligned}
$$

where

$$
\begin{aligned}
a_{\bar{q}}= & \frac{2^{\bar{q} \cdot(2 \bar{\delta}+2 \bar{a}-(3 / 2,1 / 2,1 / 2))}\left\|\Delta_{\bar{q}} w_{1}\right\|_{L^{2}}}{\left|w_{1}\right|_{2 \bar{\delta}+2 \bar{a}-(3 / 2,1 / 2,1 / 2)}} \\
& +\frac{2^{\bar{q} \cdot(2 \bar{\delta}+2 \bar{a}-(1 / 2,3 / 2,1 / 2))}\left\|\Delta_{\bar{q}} w_{2}\right\|_{L^{2}}}{\left|w_{2}\right|_{2 \bar{\delta}+2 \bar{a}-(1 / 2,3 / 2,1 / 2)}} \\
& +\frac{2^{-\bar{q} \cdot(2 \bar{\delta}+2 \bar{a}-(1 / 2,1 / 2,3 / 2))}\left\|\Delta_{\bar{q}} w_{3}\right\|_{L^{2}}}{\left|w_{3}\right|_{2 \bar{\delta}+2 \bar{a}-(1 / 2,1 / 2,3 / 2)}}
\end{aligned}
$$

so $\left\|a_{\bar{q}}(\tau)\right\|_{\ell^{2}} \leq 3$ for all $\tau$. Using this in (2.2) leads to

$$
\begin{aligned}
& \frac{d}{d t}\left\|\Delta_{\bar{q}} u_{n}\right\|_{L^{2}}^{2}+C \nu\left(4^{q_{1}}+4^{q_{2}}+4^{q_{3}}\right)\left\|\Delta_{\bar{q}} u_{n}\right\|_{L^{2}}^{2} \\
& \leq C\left(2^{-\bar{q} \cdot(2 \bar{\delta}+2 \bar{a}-(3 / 2,1 / 2,1 / 2))}+2^{-\bar{q} \cdot(2 \bar{\delta}+2 \bar{a}-(1 / 2,3 / 2,1 / 2))}\right. \\
& \left.+2^{-\bar{q} \cdot(2 \bar{\delta}+2 \bar{a}-(1 / 2,1 / 2,3 / 2))}\right) \\
& \cdot a_{\bar{q}}\left|u_{n}\right| \frac{2}{\delta}+\bar{a}|| \Delta_{\bar{q}} u_{n} \|_{L^{2}} \text {. }
\end{aligned}
$$

By Gronwall's lemma we have

$$
\begin{aligned}
& \left\|\Delta_{\bar{q}} u_{n}(t)\right\|_{L^{2}} \\
& \leq\left\|\Delta_{\bar{q}} u_{0}^{n}\right\|_{L^{2}} \exp \left(-C \nu\left(4^{q_{1}}+4^{q_{2}}+4^{q_{3}}\right) t\right) \\
& +C\left(2^{-\bar{q} \cdot(2 \bar{\delta}+2 \bar{a}-(3 / 2,1 / 2,1 / 2))}+2^{-\bar{q} \cdot(2 \bar{\delta}+2 \bar{a}-(1 / 2,3 / 2,1 / 2))}\right. \\
& \left.\quad+2^{-\bar{q} \cdot(2 \bar{\delta}+2 \bar{a}-(1 / 2,1 / 2,3 / 2))}\right) \\
& \quad \int_{0}^{t} \exp \left(-C \nu\left(4^{q_{1}}+4^{q_{2}}+4^{q_{3}}\right)(t-\tau)\right) a_{\bar{q}}(\tau)\left|u_{n}(\tau)\right|_{\bar{\delta}+\bar{a}}^{2} d \tau .
\end{aligned}
$$


Taking the $L^{4}(0, T)$ norm and using Young's inequality gives

$$
\begin{aligned}
& \left\|\Delta_{\bar{q}} u_{n}(t)\right\|_{L_{T}^{4}\left(L^{2}\right)} \\
& \leq C \nu^{-1 / 4}\left(4^{q_{1}}+4^{q_{2}}+4^{q_{3}}\right)^{-1 / 4}\left\|\Delta_{\bar{q}} u_{0}^{n}\right\|_{L^{2}} \\
& \quad \cdot\left(1-\exp \left(-C \nu T\left(4^{q_{1}}+4^{q_{2}}+4^{q_{3}}\right)\right)\right)^{1 / 4} \\
& +C\left(2^{-\bar{q} \cdot(2 \bar{\delta}+2 \bar{a}-(3 / 2,1 / 2,1 / 2))}+2^{-\bar{q} \cdot(2 \bar{\delta}+2 \bar{a}-(1 / 2,3 / 2,1 / 2))}\right. \\
& \left.\quad+2^{-\bar{q} \cdot(2 \bar{\delta}+2 \bar{a}-(1 / 2,1 / 2,3 / 2))}\right) \\
& \quad \cdot\left\|\exp \left(-C \nu\left(4^{q_{1}}+4^{q_{2}}+4^{q_{3}}\right)(\cdot)\right)\right\|_{L^{4 / 3}(0, T)}\left\|a_{\bar{q}}\left|u_{n}\right|_{\bar{\delta}+\bar{a}}^{2}\right\|_{L^{2}(0, T)} \\
& \leq C \nu^{-1 / 4}\left(4^{q_{1}}+4^{q_{2}}+4^{q_{3}}\right)^{-1 / 4}\left\|\Delta_{\bar{q}} u_{0}^{n}\right\|_{L^{2}} \\
& \quad \cdot\left(1-\exp \left(-C \nu T\left(4^{q_{1}}+4^{q_{2}}+4^{q_{3}}\right)\right)\right)^{1 / 4} \\
& +C \nu^{-3 / 4}\left(2^{-\bar{q} \cdot(2 \bar{\delta}+2 \bar{a}-(3 / 2,1 / 2,1 / 2))}+2^{-\bar{q} \cdot(2 \bar{\delta}+2 \bar{a}-(1 / 2,3 / 2,1 / 2))}\right. \\
& \left.\quad+2^{-\bar{q} \cdot(2 \bar{\delta}+2 \bar{a}-(1 / 2,1 / 2,3 / 2))}\right) \\
& \quad \cdot\left(4^{q_{1}}+4^{q_{2}}+4^{q_{3}}\right)^{-3 / 4}\left\|a_{\bar{q}}\left|u_{n}\right|_{\bar{\delta}+\bar{a}}^{2}\right\|_{L^{2}(0, T)} .
\end{aligned}
$$

Young's inequality along with relation (2.1) imply

$$
\begin{aligned}
2^{\bar{q} \cdot \bar{a}} & =2^{q_{1} a_{1}} 2^{q_{2} a_{2}} 2^{q_{3} a_{3}} \\
& \leq 2 a_{1} 2^{2 q_{1}}+2 a_{2} 2^{2 q_{2}}+2 a_{3} 2^{2 q_{3}} \\
& \leq\left(4^{q_{1}}+4^{q_{2}}+4^{q_{3}}\right) \\
2^{q_{1}\left(1-2\left(a_{1}+\delta_{1}\right) / 3\right)} 2^{q_{2}\left(1 / 3-2\left(a_{2}+\delta_{2}\right) / 3\right)} 2^{q_{3}\left(1 / 3-2\left(a_{3}+\delta_{3}\right) / 3\right)} & \leq\left(1-\frac{2}{3}\left(a_{1}+\delta_{1}\right)\right) 2^{q_{1}}+\left(\frac{1}{3}-\frac{2}{3}\left(a_{2}+\delta_{2}\right)\right) 2^{q_{2}} \\
& +\left(\frac{1}{3}-\frac{2}{3}\left(a_{3}+\delta_{3}\right)\right) 2^{q_{3}} \\
\leq & 2^{q_{1}}+2^{q_{2}}+2^{q_{3}},
\end{aligned}
$$

and two similar inequalities. Therefore

$$
\left(4^{q_{1}}+4^{q_{2}}+4^{q_{3}}\right)^{-1 / 4} \leq 2^{-\bar{q} \cdot \bar{a}},
$$




$$
\begin{aligned}
& 2^{-\bar{q} \cdot(2 \bar{\delta}+2 \bar{a}-(3 / 2,1 / 2,1 / 2))}\left(2^{q_{1}}+2^{q_{2}}+2^{q_{3}}\right)^{-3 / 2} \\
& \quad \leq 2^{-\bar{q} \cdot(2 \bar{\delta}+2 \bar{a}-(3 / 2,1 / 2,1 / 2))} 2^{-3(\bar{q} \cdot((1,1 / 3,1 / 3)-2(\bar{a}+\bar{\delta}) / 3)) / 2} \\
& \quad=2^{-\bar{q} \cdot(\bar{a}+\bar{\delta})} \\
& 2^{-\bar{q} \cdot(2 \bar{\delta}+2 \bar{a}-(1 / 2,3 / 2,1 / 2))}\left(2^{q_{1}}+2^{q_{2}}+2^{q_{3}}\right)^{-3 / 2} \\
& \quad \leq 2^{-\bar{q} \cdot(2 \bar{\delta}+2 \bar{a}-(1 / 2,3 / 2,1 / 2))} 2^{-3(\bar{q} \cdot((1 / 3,1,1 / 3)-2(\bar{a}+\bar{\delta}) / 3)) / 2} \\
& \quad=2^{-\bar{q} \cdot(\bar{a}+\bar{\delta})}, \\
& 2^{-\bar{q} \cdot(2 \bar{\delta}+2 \bar{a}-(1 / 2,1 / 2,3 / 2))}\left(2^{q_{1}}+2^{q_{2}}+2^{q_{3}}\right)^{-3 / 2} \\
& \quad \leq 2^{-\bar{q} \cdot(2 \bar{\delta}+2 \bar{a}-(1 / 2,1 / 2,3 / 2))} 2^{-3(\bar{q} \cdot((1 / 3,1 / 3,1)-2(\bar{a}+\bar{\delta}) / 3)) / 2} \\
& \quad=2^{-\bar{q} \cdot(\bar{a}+\bar{\delta})} .
\end{aligned}
$$

It follows that

$$
2^{\bar{q} \cdot(\bar{a}+\bar{\delta})}\left\|\Delta_{\bar{q}} u_{n}\right\|_{L_{T}^{4}\left(L^{2}\right)} \leq C \nu^{-1 / 4} 2^{\bar{q} \cdot \bar{\delta}}\left\|\Delta_{\bar{q}} u_{0}^{n}\right\|_{L^{2}}
$$

$$
\begin{aligned}
& \cdot\left(1-\exp \left(-C \nu T\left(4^{q_{1}}+4^{q_{2}}+4^{q_{3}}\right)\right)\right)^{1 / 4} \\
& +C \nu^{-3 / 4}\left\|a_{\bar{q}}\left|u_{n}\right|_{\bar{\delta}+\bar{a}}^{2}\right\|_{L^{2}(0, T)} .
\end{aligned}
$$

Taking the $\ell^{2}$ norm gives

$$
\begin{aligned}
& \left\|u_{n}\right\|_{H_{T}} \\
& \leq C \nu^{-1 / 4}\left\|2^{\bar{q} \cdot \bar{\delta}}\right\| \Delta_{\bar{q}} u_{0}^{n}\left\|_{L^{2}}\left(1-\exp \left(-C \nu T\left(4^{q_{1}}+4^{q_{2}}+4^{q_{3}}\right)\right)\right)^{1 / 4}\right\|_{\ell^{2}}
\end{aligned}
$$

$$
\begin{gathered}
\quad+C \nu^{-3 / 4}\left\|u_{n}(\tau)\right\|_{L^{4}(] 0, T\left[; \mathcal{H}^{\bar{\delta}+\bar{a}}\right)}^{2} \\
\leq \nu^{-1 / 4} f_{n}(T)+C \nu^{-3 / 4}\left\|u_{n}\right\|_{H_{T}}^{2},
\end{gathered}
$$

where

$$
f_{n}(T)=C\left\|2^{\bar{q} \cdot \bar{\delta}}\right\| \Delta_{\bar{q}} u_{0}^{n}\left\|_{L^{2}}\left(1-\exp \left(-C \nu T\left(4^{q_{1}}+4^{q_{2}}+4^{q_{3}}\right)\right)\right)^{1 / 4}\right\|_{\ell^{2}} .
$$

We shall need to have $f_{n}(T)$ small. In order to obtain that, we use Lebesgue's dominated convergence theorem. The particular form of $u_{0}^{n}$ implies

$$
\left\|\Delta_{\bar{q}} u_{0}^{n}\right\|_{L^{2}} \leq\left\|\Delta_{\bar{q}} S_{n} u_{0}\right\|_{L^{2}} \leq\left\|S_{n} \Delta_{\bar{q}} u_{0}\right\|_{L^{2}} \leq\left\|\Delta_{\bar{q}} u_{0}\right\|_{L^{2}}
$$


and the estimate

$2^{\bar{q} \cdot \bar{\delta}}\left\|\Delta_{\bar{q}} u_{0}^{n}\right\|_{L^{2}}\left(1-\exp \left(-C \nu T\left(4^{q_{1}}+4^{q_{2}}+4^{q_{3}}\right)\right)\right)^{1 / 4} \leq 2^{\bar{q} \cdot \bar{\delta}}\left\|\Delta_{\bar{q}} u_{0}\right\|_{L^{2}}$

fulfills the domination requirement since the right side is an $\ell^{2}$ sequence that is independent of $T$ and $n$. As for the pointwise convergence, for fixed $\bar{q}$ one has

$$
\begin{aligned}
2^{\bar{q} \cdot \bar{\delta}}\left\|\Delta_{\bar{q}} u_{0}^{n}\right\|_{L^{2}}^{2}\left(1-\exp \left(-C \nu T\left(4^{q_{1}}+4^{q_{2}}+4^{q_{3}}\right)\right)\right)^{1 / 4} & \\
& \leq\left|u_{0}\right|_{\bar{\delta}}\left(1-\exp \left(-C \nu T\left(4^{q_{1}}+4^{q_{2}}+4^{q_{3}}\right)\right)\right)^{1 / 4} \stackrel{T \rightarrow 0}{\longrightarrow} 0 .
\end{aligned}
$$

So, by Lebesgue, $\lim _{T \rightarrow 0} f_{n}(T)=0$ uniformly with respect to $n$. We choose $T$ small enough such that $f_{n}(T)<\nu /(4 C)$, where $C$ is the constant from inequality (2.5). It follows that

$$
\left\|u_{n}\right\|_{H_{T}}<\frac{\nu^{3 / 4}}{4 C}+C \nu^{-3 / 4}\left\|u_{n}\right\|_{H_{T}}^{2} .
$$

We deduce that $\left\|u_{n}\right\|_{H_{T}} \leq \nu^{3 / 4} /(2 C)$ if we take into account that $\left\|u_{n}\right\|_{H_{T}}$ is continuous in $T,\left\|u_{n}\right\|_{H_{0}}=0$ and

$$
\frac{\nu^{3 / 4}}{2 C}=\frac{\nu^{3 / 4}}{4 C}+C \nu^{-3 / 4}\left(\frac{\nu^{3 / 4}}{2 C}\right)^{2} .
$$

This allows us to take the limit and to find the existence of the solution on $[0, T]$.

Proof of the GLOBAL EXISTENCE. We start again from inequality (2.5) and we estimate $f_{n}(t) \leq C\left|u_{0}\right|_{\bar{\delta}}$. We find in the same way the existence of a solution in $L^{4}(] 0, \infty\left[; \mathcal{H}^{\bar{\delta}+\bar{a}}\right)$. Next we prove that such a solution belongs to $L^{\infty}(] 0, \infty\left[; \mathcal{H}^{\bar{\delta}}\right)$.

We start again from inequality (2.3), we apply the $L^{\infty}$ norm and making similar computations we find

$$
\begin{aligned}
& 2^{\bar{q} \cdot \bar{\delta}}\left\|\Delta_{\bar{q}} u\right\|_{L_{T}^{\infty}\left(L^{2}\right)} \\
& \quad \leq C 2^{\bar{q} \cdot \bar{\delta}}\left\|\Delta_{\bar{q}} u_{0}\right\|_{L^{2}}+C \nu^{-1 / 2}\left\|a_{\bar{q}}|u|_{\bar{\delta}+\bar{a}}^{2}\right\|_{L^{2}(0, T)} .
\end{aligned}
$$

Taking the $\ell^{2}$ norm yields

$$
\|u\|_{L^{\infty}(] 0, \infty\left[; \mathcal{H}^{\bar{\delta}}\right)} \leq\left|u_{0}\right|_{\bar{\delta}}+C \nu^{-1 / 2}\left(\|u\|_{L^{4}(] 0, \infty\left[; \mathcal{H}^{\bar{\delta}+\bar{a}}\right)}\right)^{2} .
$$


Finally, the continuity in time follows from Lebesgue's dominated convergence theorem since the map $t \longrightarrow\left\|\Delta_{\bar{q}} u_{n}\right\|_{L^{2}}$ is continous and the domination requirement is given in relations (2.6) and (2.7).

Let us now prove the uniqueness.

Theorem 2.4 (uniqueness). Let $u_{1}$ and $u_{2}$ be two solutions of (N-S) which belong to the space $L^{4}(] 0, T\left[; \mathcal{H}^{\bar{\delta}+\bar{a}}\right) \cap \mathcal{C}\left(\left[0, T\left[; \mathcal{H}^{\bar{\delta}}\right)\right.\right.$ with the same initial data in $\mathcal{H}^{\bar{\delta}}$. Then $u_{1}=u_{2}$.

Proof. We subtract the equations verified by $u_{1}$ and $u_{2}$ to obtain

$\partial_{t}\left(u_{1}-u_{2}\right)-\nu \Delta\left(u_{1}-u_{2}\right)+u_{1} \cdot \nabla\left(u_{1}-u_{2}\right)+\left(u_{1}-u_{2}\right) \nabla u_{2}=\nabla\left(p_{1}-p_{2}\right)$.

The same computations as in Theorem 2.3 yield

$$
\begin{aligned}
& \left\|u_{1}-u_{2}\right\|_{L^{4}(] 0, t\left[; \mathcal{H}^{\bar{\delta}+\bar{a}}\right)} \\
& \quad \leq C\left\|u_{1}-u_{2}\right\|_{L^{4}(] 0, t\left[; \mathcal{H}^{\bar{\delta}+\bar{a}}\right)}\left(\left\|u_{1}\right\|_{L^{4}(] 0, t\left[; \mathcal{H}^{\bar{\delta}+\bar{a}}\right)}+\left\|u_{2}\right\|_{L^{4}(] 0, t\left[; \mathcal{H}^{\bar{\delta}+\bar{a}}\right)}\right) .
\end{aligned}
$$

Thus, if $t$ is small enough, we have

$$
\left\|u_{1}-u_{2}\right\|_{L^{4}(] 0, t\left[; \mathcal{H}^{\bar{\delta}+\bar{a}}\right)} \leq \frac{1}{2}\left\|u_{1}-u_{2}\right\|_{L^{4}(] 0, t\left[; \mathcal{H}^{\bar{\delta}+\bar{a}}\right)},
$$

so we get local uniqueness that is global uniqueness, since the map $t \longrightarrow\left\|u_{1}-u_{2}\right\|_{L^{4}(] 0, t\left[; \mathcal{H}^{\bar{\delta}+\bar{a}}\right)}$ is continuous.

\section{Resolution of (N-S) in the $H B^{s_{1}, s_{2}, s_{3}}$ spaces.}

Let us introduce the spaces $H B_{T, p, s_{1}, s_{2}, s_{3}}=H B_{T, p, \bar{s}}$ defined as the closure of compactly supported smooth functions under the norm

$$
\|u\|_{H B_{T, p, \bar{s}}} \stackrel{\text { def }}{=}\|\| 2^{\bar{q} \cdot \bar{s}} \Delta_{\bar{q}} u\left\|_{L_{T}^{p}\left(L^{2}\right)}\right\|_{\ell^{2,2,1}} .
$$

As for the $\mathcal{H}^{\bar{s}}$ spaces we shall prove a theorem of global existence and uniqueness and a local existence and uniqueness one. Let $a$ and $b$ be two positive real numbers such that $a+b=1 / 2$.

Theorem 3.1 (global existence and uniqueness). There exists $C>0$ such that if $\operatorname{div} u_{0}=0, u_{0} \in H B^{0,0,1 / 2}$ and $|u|_{H B^{0,0,1 / 2}}<C \nu$ then the $(\mathrm{N}-\mathrm{S})$ equations have a unique global solution which belongs to

$$
H B_{\infty, 4, a, b, 1 / 2} \cap L^{\infty}(] 0, \infty\left[; H B^{0,0,1 / 2}\right) \cap \mathcal{C}\left(\left[0, \infty\left[; H B^{0,0,1 / 2}\right) .\right.\right.
$$


Theorem 3.2 (local existence and uniqueness). If $\operatorname{div} u_{0}=0$ and $u_{0} \in H B^{0,0,1 / 2}$ then there exist $T>0$ and a unique solution of $(\mathrm{N}-\mathrm{S})$ on $[0, T]$ which belongs to $H B_{T, 4, a, b, 1 / 2} \cap \mathcal{C}\left(\left[0, T\left[; H B^{0,0,1 / 2}\right)\right.\right.$.

REMARK. We have $H B_{T, 4, a, b, 1 / 2} \hookrightarrow L^{4}(] 0, \infty\left[; H B^{a, b, 1 / 2}\right)$.

Indeed, Remark 1.1 implies

$$
\begin{aligned}
\|u\|_{L^{4}(] 0, \infty\left[; H B^{a, b, 1 / 2}\right)} & =\|\| 2^{q_{1} a+q_{2} b+q_{3} / 2}\left\|\Delta_{\bar{q}} u\right\|_{L^{2}}\left\|_{\ell^{2,2,1}}\right\|_{L^{4}} \\
& \leq\|\| 2^{q_{1} a+q_{2} b+q_{3} / 2}\left\|\Delta_{\bar{q}} u\right\|_{L^{2}}\left\|_{L^{4}}\right\|_{\ell^{2,2,1}} \\
& =\|u\|_{H B_{T, 4, a, b / 2}} .
\end{aligned}
$$

We first prove

Lemma 3.1. Let $s_{i}<1 / 2, t_{i}<1 / 2, s_{i}+t_{i}>0$, for all $i \in\{1,2\}$, $s_{3} \leq 1 / 2, t_{3} \leq 1 / 2, s_{3}+t_{3}>0$ and $p, q \geq 1, r=p q /(p+q) \geq 1$. Then

$$
\|u v\|_{H B_{T, r, \bar{s}+\bar{t}-(1 / 2,1 / 2,1 / 2)}} \leq\|u\|_{H B_{T, p, \bar{s}}}\|v\|_{H B_{T, q, \bar{t}}} .
$$

Proof. We shall copy the proof of Theorem 1.2 and prove this lemma for each of the 27 terms of the Littlewood-Paley decomposition. Let us take, for instance, the $T^{1} R^{2} \widetilde{T}^{3}$ term. We start again from inequality (1.4)

$$
\begin{gathered}
\left\|\Delta_{\bar{q}} w_{\bar{p}}^{i}(t)\right\|_{L^{2}} \leq 2^{q_{2} / 2} \sum_{\substack{r_{1} \leq p_{1}-2 \\
r_{3} \leq p_{3}-2}} 2^{r_{1} / 2+r_{3} / 2}\left\|\Delta_{r_{1}}^{1} \Delta_{p_{2}}^{2} \Delta_{p_{3}}^{3} u(t)\right\|_{L^{2}} \\
\cdot\left\|\Delta_{p_{1}}^{1} \Delta_{p_{2}-i}^{2} \Delta_{r_{3}}^{3} v(t)\right\|_{L^{2}} .
\end{gathered}
$$

Taking the $L^{r}(0, T)$ norm and applying Hölder's inequality gives

$$
\begin{gathered}
\left\|\Delta_{\bar{q}} w_{\bar{p}}^{i}(t)\right\|_{L_{T}^{r}\left(L^{2}\right)} \leq 2^{q_{2} / 2} \sum_{\substack{r_{1} \leq p_{1}-2 \\
r_{3} \leq p_{3}-2}} 2^{r_{1} / 2+r_{3} / 2}\left\|\Delta_{r_{1}}^{1} \Delta_{p_{2}}^{2} \Delta_{p_{3}}^{3} u(t)\right\|_{L_{T}^{p}\left(L^{2}\right)} \\
\qquad\left\|\Delta_{p_{1}}^{1} \Delta_{p_{2}-i}^{2} \Delta_{r_{3}}^{3} v(t)\right\|_{L_{T}^{q}\left(L^{2}\right)}
\end{gathered}
$$

If we define

$$
A_{\bar{q}}=2^{\bar{q} \cdot \bar{s}}\left\|\Delta_{\bar{q}} u\right\|_{L_{T}^{p}\left(L^{2}\right)}
$$


and

$$
B_{\bar{q}}=2^{\bar{q} \cdot \bar{t}}\left\|\Delta_{\bar{q}} u\right\|_{L_{T}^{q}\left(L^{2}\right)},
$$

it follows that

$$
\begin{aligned}
2^{\bar{q} \cdot(\bar{s}+\bar{t}-(1 / 2,1 / 2,1 / 2))}\left\|\Delta_{\bar{q}} w \frac{i}{p}(t)\right\|_{L_{T}^{r}\left(L^{2}\right)} & \\
\leq & C 2^{q_{1}\left(s_{1}-1 / 2\right)+\left(s_{2}+t_{2}\right)\left(q_{2}-p_{2}\right)+q_{3}\left(t_{3}-1 / 2\right)} \\
& \cdot \sum_{\substack{r_{1} \leq p_{1}-2 \\
r_{3} \leq p_{3}-2}} 2^{r_{1}\left(1 / 2-s_{1}\right)+r_{3}\left(1 / 2-t_{3}\right)} A_{r_{1}, p_{2}, p_{3}} B_{p_{1}, p_{2}-i, r_{3}} .
\end{aligned}
$$

This inequality is entirely similar to (1.5) so the proof continues in exactly the same way we did after that inequality.

Proof of the LOCAL EXISTEnCE. It is obvious that if $\bar{\delta}=(0,0,1 / 2)$ and $a_{1}=a, a_{2}=b, a_{3}=0$ then hypothesis (2.1) is verified excepted for the condition $\delta_{3}+a_{3}<1 / 2$. This is precisely where we use that $B_{2,1}^{1 / 2}(\mathbb{R})$ is an algebra. Hence, we can follow the same line of proof as in Theorem 2.3, replacing the $\ell^{2}$ norms by the $\ell^{2,2,1}$ norms and the $\mathcal{H}^{\bar{s}}$ spaces with the $H B^{\bar{s}}$ spaces. There is one fact which doesn't allow us to give an identical proof: the deduction of inequality (2.5) from inequality (2.4) which is not possible because the switch of the $L^{2}$ and $\ell^{2,2,1}$ norms yields an inequality in the opposite sens of the wanted one. To avoid that we have to give up the estimate

$$
\begin{aligned}
\left\|\Delta_{\bar{q}}(u \nabla u)\right\|_{L^{2}} \leq C & \left(2^{-\bar{q} \cdot(2 \bar{\delta}+2 \bar{a}-(3 / 2,1 / 2,1 / 2))}+2^{-\bar{q} \cdot(2 \bar{\delta}+2 \bar{a}-(1 / 2,3 / 2,1 / 2))}\right. \\
& \left.+2^{-\bar{q} \cdot(2 \bar{\delta}+2 \bar{a}-(1 / 2,1 / 2,3 / 2))}\right) a_{\bar{q}}|u|_{1 / 2+\delta, 1 / 2-\delta}^{2}
\end{aligned}
$$

and to use, for the deduction of inequality (2.5), Lemma 3.1. As in Theorem 2.3 we find the following inequality

$$
\begin{aligned}
\frac{d}{d t}\left\|\Delta_{\bar{q}} u_{n}\right\|_{L^{2}}^{2}+C \nu\left(4^{q_{1}}+4^{q_{2}}+4^{q_{3}}\right)\left\|\Delta_{\bar{q}} u_{n}\right\|_{L^{2}}^{2} & \\
& \leq\left\|\Delta_{\bar{q}}\left(u_{n} \nabla u_{n}\right)\right\|_{L^{2}}\left\|\Delta_{\bar{q}} u_{n}\right\|_{L^{2}} .
\end{aligned}
$$

Gronwall's lemma implies

$$
\begin{aligned}
& \left\|\Delta_{\bar{q}} u_{n}(t)\right\|_{L^{2}} \\
& \leq\left\|\Delta_{\bar{q}} u_{0}^{n}\right\|_{L^{2}} \exp \left(-C \nu\left(4^{q_{1}}+4^{q_{2}}+4^{q_{3}}\right) t\right) \\
& \quad+C\left(\exp \left(-C \nu\left(4^{q_{1}}+4^{q_{2}}+4^{q_{3}}\right)(\cdot)\right)\left(\left\|\Delta_{\bar{q}}\left(u_{n}(\cdot) \nabla u_{n}(\cdot)\right)\right\|_{L^{2}}\right)\right)(t) .
\end{aligned}
$$


Taking the $L^{4}(0, T)$ norm and using Young's inequality gives

$$
\begin{aligned}
&\left\|\Delta_{\bar{q}} u_{n}(t)\right\|_{L_{T}^{4}\left(L^{2}\right)} \\
& \leq C \nu^{-1 / 4}\left(4^{q_{1}}+4^{q_{2}}+4^{q_{3}}\right)^{-1 / 4}\left\|\Delta_{\bar{q}} u_{0}^{n}\right\|_{L^{2}} \\
& \cdot\left(1-\exp \left(-C \nu T\left(4^{q_{1}}+4^{q_{2}}+4^{q_{3}}\right)\right)\right)^{1 / 4} \\
&+C\left\|\exp \left(-C \nu\left(4^{q_{1}}+4^{q_{2}}+4^{q_{3}}\right)(\cdot)\right)\right\|_{L^{4 / 3}(0, T)}\left\|\Delta_{\bar{q}}\left(u_{n} \nabla u_{n}\right)\right\|_{L_{T}^{2}\left(L^{2}\right)} \\
& \leq C \nu^{-1 / 4}\left(4^{q_{1}}+4^{q_{2}}+4^{q_{3}}\right)^{-1 / 4}\left\|\Delta_{\bar{q}} u_{0}^{n}\right\|_{L^{2}} \\
& \cdot\left(1-\exp \left(-C \nu T\left(4^{q_{1}}+4^{q_{2}}+4^{q_{3}}\right)\right)\right)^{1 / 4} \\
&+C \nu^{-3 / 4}\left(4^{q_{1}}+4^{q_{2}}+4^{q_{3}}\right)^{-3 / 4}\left\|\Delta_{\bar{q}}\left(u_{n} \nabla u_{n}\right)\right\|_{L_{T}^{2}\left(L^{2}\right)} .
\end{aligned}
$$

Again by Young's inequality we have

$$
\left(4^{q_{1}}+4^{q_{2}}+4^{q_{3}}\right)^{-1 / 4} \leq 2^{q_{3} / 2} 2^{-\bar{q} \cdot(a, b, 1 / 2)} .
$$

It follows that

$$
\begin{aligned}
2^{\bar{q} \cdot(a, b, 1 / 2)}\left\|\Delta_{\bar{q}} u_{n}(t)\right\|_{L_{T}^{4}\left(L^{2}\right)} \\
\leq C \nu^{-1 / 4} 2^{q_{3} / 2}\left\|\Delta_{\bar{q}} u_{0}^{n}\right\|_{L^{2}} \\
\quad \cdot\left(1-\exp \left(-C \nu T\left(4^{q_{1}}+4^{q_{2}}+4^{q_{3}}\right)\right)\right)^{1 / 4} \\
+C \nu^{-3 / 4}\left(4^{q_{1}}+4^{q_{2}}+4^{q_{3}}\right)^{-3 / 4} 2^{\bar{q} \cdot(a, b, 1 / 2)} \\
\quad \cdot\left\|\Delta_{\bar{q}}\left(u_{n} \nabla u_{n}\right)\right\|_{L_{T}^{2}\left(L^{2}\right)} \cdot
\end{aligned}
$$

Now we use the Lemma 3.1 to deduce that

$$
\begin{aligned}
&\left\|\Delta_{\bar{q}}\left(u_{n} \nabla u_{n}\right)\right\|_{L_{T}^{2}\left(L^{2}\right)} \\
&=\| \Delta_{\bar{q}} \operatorname{div}\left(u_{n} \otimes u_{n}\right) \|_{L_{T}^{2}\left(L^{2}\right)} \\
& \leq C c_{\bar{q}}\left(2^{-\bar{q} \cdot(2 a-3 / 2,2 b-1 / 2,1 / 2)}+2^{-\bar{q} \cdot(2 a-1 / 2,2 b-3 / 2,1 / 2)}\right. \\
&\left.\quad+2^{-\bar{q} \cdot(2 a-1 / 2,2 b-1 / 2,-1 / 2)}\right)\left\|u_{n}\right\|_{H B_{T, 4, a, b, 1 / 2}}^{2},
\end{aligned}
$$

where $\left\|c_{\bar{q}}\right\|_{\ell^{2,2,1}}=1$. Young's inequality implies

$$
\begin{gathered}
\left(4^{q_{1}}+4^{q_{2}}+4^{q_{3}}\right)^{-3 / 4} 2^{\bar{q} \cdot(a, b, 1 / 2)} 2^{-\bar{q} \cdot(2 a-3 / 2,2 b-1 / 2,1 / 2)} \leq 1, \\
\left(4^{q_{1}}+4^{q_{2}}+4^{q_{3}}\right)^{-3 / 4} 2^{\bar{q} \cdot(a, b, 1 / 2)} 2^{-\bar{q} \cdot(2 a-1 / 2,2 b-3 / 2,1 / 2)} \leq 1, \\
\left(4^{q_{1}}+4^{q_{2}}+4^{q_{3}}\right)^{-3 / 4} 2^{\bar{q} \cdot(a, b, 1 / 2)} 2^{-\bar{q} \cdot(2 a-1 / 2,2 b-1 / 2,-1 / 2)} \leq 1 .
\end{gathered}
$$


Hence inequality (3.1) may be written as

$$
\begin{aligned}
& 2^{\bar{q} \cdot(a, b, 1 / 2)}\left\|\Delta_{\bar{q}} u_{n}\right\|_{L_{T}^{4}\left(L^{2}\right)} \\
& \leq C \nu^{-1 / 4} 2^{q_{3} / 2}\left\|\Delta_{\bar{q}} u_{0}^{n}\right\|_{L^{2}}\left(1-\exp \left(-C \nu T\left(4^{q_{1}}+4^{q_{2}}+4^{q_{3}}\right)\right)\right)^{1 / 4} \\
& \quad+C \nu^{-3 / 4} c_{\bar{q}}\left\|u_{n}\right\|_{H B_{T, 4, a, b, 1 / 2}}^{2} \cdot
\end{aligned}
$$

Taking the $\ell^{2,2,1}$ norm gives

$$
\begin{aligned}
& \left\|u_{n}\right\|_{H B_{T, 4, a, b, 1 / 2}} \\
& \leq C\left\|2^{q_{3} / 2}\right\| \Delta_{\bar{q}} u_{0}\left\|_{L^{2}}\left(1-\exp \left(-C \nu T\left(4^{q_{1}}+4^{q_{2}}+4^{q_{3}}\right)\right)\right)^{1 / 4}\right\|_{\ell^{2,2,1}} \\
& \quad+C\left\|u_{n}(\tau)\right\|_{H B_{T, 4, a, b, 1 / 2}}^{2} \\
& \leq g_{n}(T)+C\left\|u_{n}\right\|_{H B_{T, 4, a, b, 1 / 2}}^{2}
\end{aligned}
$$

where

$g_{n}(t)=C\left\|2^{q_{3} / 2}\right\| \Delta_{\bar{q}} u_{0}\left\|_{L^{2}}\left(1-\exp \left(-C \nu T\left(4^{q_{1}}+4^{q_{2}}+4^{q_{3}}\right)\right)\right)^{1 / 4}\right\|_{\ell^{2,2,1}}$.

We conclude as in Theorem 2.3. The fact that $u \in \mathcal{C}\left(\left[0, T\left[; H B^{0,0,1 / 2}\right)\right.\right.$ is proved as in the case of $\mathcal{H}^{\bar{s}}$ spaces.

Proof of the global existence. Same proof as above by estimating

$$
g_{n}(t) \leq C\left|u_{0}\right|_{H B^{0,0,1 / 2}} .
$$

The uniqueness theorem is also similar to the one of the case $\mathcal{H}^{\bar{s}}$.

Theorem 3.3 (uniqueness). Let $u_{1}$ and $u_{2}$ be two solutions of (N-S) which belong to $H B_{T, 4, a, b, 1 / 2} \cap \mathcal{C}\left(\left[0, T\left[; H B^{0,0,1 / 2}\right)\right.\right.$ with the same initial data in $H B^{0,0,1 / 2}$. Then $u_{1}=u_{2}$.

Proof. Making the same computations as in Theorem 2.4, replacing the $\ell^{2}$ norms with the $\ell^{2,2,1}$ norms and using Lemma 3.1 as shown above we find

$$
\begin{aligned}
& \left\|u_{1}-u_{2}\right\|_{H B_{t, 4, a, b, 1 / 2}} \\
& \quad \leq C\left\|u_{1}-u_{2}\right\|_{H B_{t, 4, a, b, 1 / 2}}\left(\left\|u_{1}\right\|_{H B_{t, 4, a, b, 1 / 2}}+\left\|u_{2}\right\|_{H B_{t, 4, a, b, 1 / 2}}\right) .
\end{aligned}
$$

We conclude as in Theorem 2.4. 


\section{Some imbeddings and nonimbeddings.}

In this section we prove some imbeddings and some nonimbeddings which are used to compare the results from the previous sections with the results already known. We recall that one can solve (N-S) in the spaces $H^{1 / 2}, B_{p, \infty}^{-1+3 / p}, 2 \leq p<\infty$ (see [2], [3], [7], [9]) and it seems very difficult to do it in $C^{-1}\left(\|u\|_{B_{p, q}^{s}} \stackrel{\text { def }}{=}\left\|2^{i s}\right\| \Delta_{i} u\left\|_{L^{p}}\right\|_{\ell^{q}}\right.$ and $\left.C^{-1}=B_{\infty, \infty}^{-1}\right)$. It is also proved by H. Kozono and M. Yamazaki in [8] that one can solve (N-S) in the homogeneous spaces $\mathcal{N}_{p, q, \infty}^{3 / p-1}, 1 \leq q \leq p<\infty, p>3$, where $\mathcal{N}_{p, q, r}^{s}$ is defined to be the closure of the compactly supported smooth functions under the norm

$$
\|u\|_{\mathcal{N}_{p, q, r}^{s}}=\left\|2^{s j} \sup _{x_{0} \in \mathbb{R}^{3}} \sup _{R>0} R^{3 / p-3 / q}\right\| \Delta_{j} u\left\|_{L^{q}\left(B\left(x_{0}, R\right)\right)}\right\|_{\ell^{r}},
$$

where $B\left(x_{0}, R\right)$ denotes the closed ball in $\mathbb{R}^{3}$ with center $x_{0}$ and radius $R$. Let us remark that $B_{p, r}^{s}=\mathcal{N}_{p, p, r}^{s}$. We can prove the following proposition:

Proposition 4.1. i) If $\delta_{1}+\delta_{2}+\delta_{3}=1 / 2,-1 / 2<\delta_{i}<1 / 2$ for all $i \in\{1,2,3\}$ and $p>\max _{1 \leq i \leq 3}\left(2 /\left(1-2 \delta_{i}\right)\right)$ then

$$
\mathcal{H}^{\bar{\delta}} \hookrightarrow B_{p, \infty}^{-1+3 / p} \hookrightarrow C^{-1}
$$

ii) $L^{2} \cap \mathcal{H}^{0,0,1 / 2} \not \subset C^{-1}$.

iii) If $1 \leq q \leq p<3 q / 2, p>3$, then $H B^{0,0,1 / 2} \not \subset \mathcal{N}_{p, q, \infty}^{3 / p-1}$ hence $H B^{0,0,1 / 2} \not \subset B_{p, \infty}^{-1+3 / p}$ for all $2 \leq p<\infty$.

iv) $H B^{0,0,1 / 2} \hookrightarrow C^{-1}$.

Property i) shows that solutions of (N-S) were already constructed by M. Cannone [2], F. Planchon [9] and H. Kozono, M. Yamazaki [8]. Property ii) suggests that the space $\mathcal{H}^{0,0,1 / 2}$ is very interesting as space of initial data; unfortunately we cannot include it in our results. Finally, property iii) shows that $H B^{0,0,1 / 2}$ is not included in the space considered by H. Kozono and M. Yamazaki at least for some $p$ and $q$; it implies that it is not included in any of the spaces used by M. Cannone and F. Planchon. The author doesn't know if the non-imbedding of iii) still holds for the other values of $p$ and $q$. 
Proof of Proposition 4.1. i) First we remark that if $s<0$, then we can replace $\Delta_{i}$ with $S_{i}$ in the definition of the $B_{p, \infty}^{s}$ space. By Lemma 1.1 we have

$$
\begin{aligned}
& 2^{q(-1+3 / p)}\left\|S_{q} u\right\|_{L^{p}} \\
& \leq C 2^{q(-1+3 / p)} \sum_{\substack{q_{1} \leq q \\
q_{2} \leq q \\
q_{3} \leq q}}\left\|\Delta_{\bar{q}} u\right\|_{L^{p}} \\
& \leq C 2^{q(-1+3 / p)} \sum_{q_{1} \leq q} 2^{q_{1}(1 / 2-1 / p)+q_{2}(1 / 2-1 / p)+q_{3}(1 / 2-1 / p)}\left\|\Delta_{\bar{q}} u\right\|_{L^{2}} \\
& \begin{array}{l}
q_{1} \leq q \\
q_{2} \leq q
\end{array} \\
& \leq C 2^{q(-1+3 / p)} \sum_{\substack{q_{1} \leq q \\
q_{2} \leq q \\
q_{3} \leq q}} 2^{q_{1}\left(1 / 2-1 / p-\delta_{1}\right)+q_{2}\left(1 / 2-1 / p-\delta_{2}\right)+q_{3}\left(1 / 2-1 / p-\delta_{3}\right)} \\
& \cdot 2^{\bar{q} \cdot \bar{\delta}}\left\|\Delta_{\bar{q}} u\right\|_{L^{2}} \\
& \leq C 2^{q(-1+3 / p)} \sum_{\substack{q_{1} \leq q \\
q_{2} \leq q \\
q_{3} \leq q}} 2^{q_{1}\left(1 / 2-1 / p-\delta_{1}\right)+q_{2}\left(1 / 2-1 / p-\delta_{2}\right)+q_{3}\left(1 / 2-1 / p-\delta_{3}\right)}|u|_{\delta} .
\end{aligned}
$$

As $1 / 2-1 / p-\delta_{i}>0$ for any $i \in\{1,2,3\}$, one deduces

$$
\begin{aligned}
& \sum_{\substack{q_{1} \leq q \\
q_{2} \leq q \\
q_{3} \leq q}} 2^{q_{1}\left(1 / 2-1 / p-\delta_{1}\right)+q_{2}\left(1 / 2-1 / p-\delta_{2}\right)+q_{3}\left(1 / 2-1 / p-\delta_{3}\right)} \\
& \leq C 2^{q\left(3 / 2-3 / p-\delta_{1}-\delta_{2}-\delta_{3}\right)} \\
& =C 2^{q(1-3 / p)} .
\end{aligned}
$$

Hence $\|u\|_{B_{p, \infty}^{-1+3 / p}} \leq C|u|_{\bar{\delta}}$ and the first imbedding is proved. In order to obtain the second imbedding it is enough to apply the classical Littlewood-Paley inequality

$$
\left\|\Delta_{q} u\right\|_{L^{\infty}} \leq 2^{3 q / p}\left\|\Delta_{q} u\right\|_{L^{p}},
$$

to multiply by $2^{-q}$ and to take the upper bound on $q$.

ii) As $L^{2} \cap \mathcal{H}^{0,0,1 / 2}$ and $C^{-1}$ are distribution spaces, the closed graph theorem shows that it is enough to prove $L^{2} \cap \mathcal{H}^{0,0,1 / 2} \hookrightarrow C^{-1}$. Assume by absurd that $L^{2} \cap \mathcal{H}^{0,0,1 / 2} \hookrightarrow C^{-1}$. Then

$$
2^{-q}\left\|S_{q} u\right\|_{L^{\infty}} \leq C\|u\|_{L^{2} \cap \mathcal{H}^{0,0,1 / 2}}, \quad \text { for all } q .
$$


We choose $u=f \otimes g$ where $f: \mathbb{R}^{2} \longrightarrow \mathbb{C}, g: \mathbb{R} \longrightarrow \mathbb{C}$. It is obvious that $S_{q} u=S_{q} f \otimes S_{q} g$ and $\|u\|_{L^{2} \cap \mathcal{H}^{0,0,1 / 2}}=\|f\|_{L^{2}}\|g\|_{H^{1 / 2}}$, where in $S_{q} f, S_{q}$ is the $2 \mathrm{D} S_{q}$ and in $S_{q} g, S_{q}$ is the 1D $S_{q}$. Hence

$$
2^{-q}\left\|S_{q} f\right\|_{L^{\infty}}\left\|S_{q} g\right\|_{L^{\infty}} \leq C\|f\|_{L^{2}}\|g\|_{H^{1 / 2}}, \quad \text { for all } q .
$$

For each fixed $q$ we use the function $f_{q}(x)=f_{0}\left(2^{q} x\right)$, where $f_{0}$ is chosen with supp $\widehat{f}_{0}$ sufficiently small to get $S_{q} f_{q}=f_{q}$, that gives $\left\|S_{q} f_{q}\right\|_{L^{\infty}}=\left\|f_{q}\right\|_{L^{\infty}}=\left\|f_{0}\right\|_{L^{\infty}}$ and $\left\|f_{q}\right\|_{L^{2}}=2^{-q}\left\|f_{0}\right\|_{L^{2}}$ since we work in two dimensions. Therefore, it comes from relation (4.1)

$$
\left\|S_{q} g\right\|_{L^{\infty}} \leq C\|g\|_{H^{1 / 2}},
$$

that is $H^{1 / 2}(\mathbb{R}) \subset L^{\infty}$ which is false.

iii) As above we assume by absurd that $H B^{0,0,1 / 2} \hookrightarrow \mathcal{N}_{p, q, \infty}^{3 / p-1}$ and we remark that if $s<0$, then we can replace $\Delta_{j}$ with $S_{j}$ in the definition of the norm of the space $\mathcal{N}_{p, q, \infty}^{s}$.

Again, we choose $u=f \otimes g$ where $f: \mathbb{R}^{2} \longrightarrow \mathbb{C}, g: \mathbb{R} \longrightarrow \mathbb{C}$. It is not difficult to see that the norm

$$
\sup _{x_{0} \in \mathbb{R}^{3}} \sup _{R>0} R^{3 / p-3 / q}\|f \otimes g\|_{L^{q}\left(B\left(x_{0}, R\right)\right)}
$$

is equivalent to the norm

$$
\sup _{x_{0} \in \mathbb{R}^{3}} \sup _{R>0} R^{3 / p-3 / q}\|f\|_{L^{q}\left(B^{2}\left(x_{0}^{\prime}, R\right)\right)}\|g\|_{L^{q}\left(B^{1}\left(x_{0}^{3}, R\right)\right)},
$$

where $B^{1}$ and $B^{2}$ denote the one-dimensional, respectively two-dimensional balls. This is done by including a cube of size $R$ into the ball $B\left(x_{0}, R\right)$, applying Fubini's theorem, then including balls of radius $R / 2$ into the one-dimensional and the two-dimensional cubes of size $R$ and finally taking the upper bound on $R$.

It follows that

$$
\begin{array}{r}
2^{j(-1+3 / p)} \sup _{x_{0} \in \mathbb{R}^{3}} \sup _{R>0} R^{3 / p-3 / q}\left\|S_{j} f\right\|_{L^{q}\left(B^{2}\left(x_{0}^{\prime}, R\right)\right)} \\
\quad\left\|S_{j} g\right\|_{L^{q}\left(B^{1}\left(x_{0}^{3}, R\right)\right)} \\
\leq C\|f\|_{L^{2}}\|g\|_{B_{2,1}^{1 / 2}},
\end{array}
$$

for all $j$, where the constant $C$ does not depend on $j$. Choosing $x_{0}=0$ yields

$$
\begin{aligned}
2^{j(-1+3 / p)} \sup _{R>0} R^{3 / p-3 / q}\left\|S_{j} f\right\|_{L^{q}\left(B^{2}(0, R)\right)} & \left\|S_{j} g\right\|_{L^{q}\left(B^{1}(0, R)\right)} \\
& \leq C\|f\|_{L^{2}}\|g\|_{B_{2,1}^{1 / 2}},
\end{aligned}
$$


for all $j$. Now we fix $j$ and we choose $f_{j}(x)=f_{0}\left(2^{j} x\right)$, the same example as above. We also choose $g$ to be a function whose Fourier transform is a compactly supported smooth function. This implies that $S_{j} f_{j}=f_{j}$ and $S_{j} g=g$ for $j$ large enough. Moreover, we have that

$$
\left\|S_{j} f_{j}\right\|_{L^{q}\left(B^{2}(0, R)\right)}=\left\|f_{j}\right\|_{L^{q}\left(B^{2}(0, R)\right)}=2^{-2 j / q}\left\|f_{0}\right\|_{L^{q}\left(B^{2}\left(0,2^{j} R\right)\right)},
$$

and

$$
\left\|f_{j}\right\|_{L^{2}}=2^{-j}\left\|f_{0}\right\|_{L^{2}} \text {. }
$$

It follows that, for $j$ large enough, we have

$$
\begin{array}{r}
2^{j(3 / p-2 / q)} \sup _{R>0} R^{3 / p-3 / q}\left\|f_{0}\right\|_{L^{q}\left(B^{2}\left(0,2^{j} R\right)\right)}\|g\|_{L^{q}\left(B^{1}(0, R)\right)} \\
\quad \leq C\left\|f_{0}\right\|_{L^{2}}\|g\|_{B_{2,1}^{1 / 2}},
\end{array}
$$

which implies

$$
\begin{aligned}
2^{j(3 / p-2 / q)} \sup _{R>0} R^{3 / p-3 / q}\left\|f_{0}\right\|_{L^{q}\left(B^{2}(0, R)\right)}\|g\|_{L^{q}\left(B^{1}(0, R)\right)} & \\
& \leq C\left\|f_{0}\right\|_{L^{2}}\|g\|_{B_{2,1}^{1 / 2}},
\end{aligned}
$$

for all $j>j_{0}$. Taking the limit on $j \longrightarrow \infty$ gives a contradiction.

iv) We write

$$
\begin{aligned}
2^{-q}\left\|S_{q} u\right\|_{L^{\infty}} & \leq 2^{-q} \sum_{\substack{q_{1} \leq q \\
q_{2} \leq q \\
q_{3} \leq q}}\left\|\Delta_{\bar{q}} u\right\|_{L^{\infty}} \\
& \leq 2^{-q} \sum_{\substack{q_{1} \leq q \\
q_{2} \leq q \\
q_{3} \leq q}} 2^{q_{1} / 2+q_{2} / 2+q_{3} / 2}\left\|\Delta_{\bar{q}} u\right\|_{L^{2}} \\
& \leq 2^{-q} \sum_{\substack{q_{1} \leq q \\
q_{2} \leq q}} 2^{q_{1} / 2+q_{2} / 2}\left\|2^{q_{3} / 2}\right\| \Delta_{\bar{q}} u\left\|_{L^{2}}\right\|_{\ell_{q_{3}}^{1}} \\
& \leq\left\|2^{q_{3} / 2}\right\| \Delta_{\bar{q}} u\left\|_{L^{2}}\right\|_{\ell^{\infty, \infty, 1}} \\
& \leq\left\|2^{q_{3} / 2}\right\| \Delta_{\bar{q}} u\left\|_{L^{2}}\right\|_{\ell^{2,2,1}} \\
& =\|u\|_{H B^{0,0,1 / 2}} .
\end{aligned}
$$

This completes the proof. 
One could ask whether the divergence free condition has an influence on the choice of the spaces where we can take the initial data or not. The answer is negative because, if we look to the proofs above, we see that the scalar counterexamples $f$ we deduce have the property that $\partial_{1} f$ and $\partial_{2} f$ are again good counterexamples (differentiating $f_{0}$ only diminishes the support of its Fourier transform), so we can take as initial data $u_{0}=\left(\partial_{2} f,-\partial_{1} f, 0\right)$.

\section{Appendix.}

In this paragraph we show how a general $d$-dimensional hyperbolic symmetric system can be solved in $B_{2,1}^{1+d / 2}\left(\mathbb{R}^{d}\right)$. By general hyperbolic symmetric system we mean a system of the form

$$
\left\{\begin{array}{l}
\partial_{t} U+A(U) \cdot \nabla U=0, \\
\left.U\right|_{t=0}=U_{0},
\end{array}\right.
$$

where

$$
A(U)=\left(A_{j}(U)\right)_{1 \leq j \leq d}
$$

and, for all $j, A_{j}(U)$ is a symmetric smooth globally Lipschitz matrix and $U$ is a time dependent vector field in $\mathbb{R}^{d}$.

Proposition. Assume that $U_{0} \in L^{2} \cap B_{2,1}^{1+d / 2}$. Then there exist a time $T$ and a unique solution of $(\mathrm{S})$ on $[0, T]$ in the space $L^{\infty}(] 0, T\left[; B_{2,1}^{1+d / 2}\right)$. Moreover, there exists a constant $C>0$ such that the maximal time existence of such a solution may be bounded from below by

$$
T>\frac{C}{\left\|U_{0}\right\|_{B_{2,1}^{1+2}}} .
$$

Proof. The proof relies on the fact that $B_{2,1}^{d / 2}$ is imbedded in $L^{\infty}$ and on the following estimate:

Lemma. For all vector fields $U$ in $B_{2,1}^{1+d / 2}$ there exists a sequence $\left\{c_{q}\right\}_{q \in \mathbb{N}}$ such that

$$
\left|\left\langle\Delta_{q}(A(U) \cdot \nabla U) \mid \Delta_{q} U\right\rangle\right| \leq C 2^{-q(d / 2+1)} c_{q}\left\|\Delta_{q} U\right\|_{L^{2}}\|U\|_{B_{2,1}^{1+d / 2}}\|\nabla U\|_{L^{\infty}}
$$


where

$$
\sum_{q} c_{q}=1
$$

This lemma is well-known in the case of the Sobolev spaces and the extension to the Besov spaces is simple. Decomposing the product $A(U) \cdot \nabla U$ in the usual sum of two paraproducts and a remainder, using the classical product theorem for Besov spaces, we see that the only term where a critical case appears is

$$
\left\langle\Delta_{q}\left(T_{A(U)} \nabla U\right) \mid \Delta_{q} U\right\rangle
$$

Some easy computations done integrating by parts show that

$$
\begin{aligned}
\left\langle\Delta_{q}\left(T_{A(U)} \nabla U\right) \mid \Delta_{q} U\right\rangle & \\
= & \sum_{p, j}\left\langle\left[\Delta_{q}, S_{p-1} A_{j}(U)\right] \partial_{j} \Delta_{p} U, \Delta_{q} U\right\rangle \\
& -\frac{1}{2} \sum_{p} S_{p-1} \operatorname{div} A(U) \Delta_{q} \Delta_{p} U \Delta_{q} U \\
& -\frac{1}{2} \sum_{p, p^{\prime}, j}\left(S_{p-1}-S_{p^{\prime}-1}\right) A_{j}(U) \Delta_{q} \Delta_{p} U \partial_{j} \Delta_{q} \Delta_{p^{\prime}} U .
\end{aligned}
$$

The last two terms are very easy to estimate, we need only to apply the definition of the Besov spaces. The first term is estimated by remarking that $\Delta_{q}$ is an operator of convolution with the function

$$
2^{q d} h\left(2^{q} \cdot\right)
$$

where $h=\mathcal{F}^{-1} \phi$. Therefore

$$
\begin{aligned}
& {\left[S_{p-1} A_{j}(U), \Delta_{q}\right] a(x)} \\
& \quad=2^{q d} \int\left(S_{p-1} A_{j}(U)(x)-S_{p-1} A_{j}(U)(y)\right) h\left(2^{q}(x-y)\right) a(y) d y .
\end{aligned}
$$

Hence

$$
\left|\left[S_{p-1} A_{j}(U), \Delta_{q}\right] a(x)\right| \leq C 2^{q(d-1)}\|\nabla U\|_{L^{\infty}}|y h|\left(2^{q} \cdot\right)|a| .
$$

Young's inequality now gives

$$
\left\|\left[S_{p-1} A_{j}(U), \Delta_{q}\right] a(x)\right\|_{L^{2}} \leq C 2^{-q}\|\nabla U\|_{L^{\infty}}\|a\|_{L^{2}} .
$$


This proves the lemma.

We return to the proof of the proposition. We apply $\Delta_{q}$ to $(\mathrm{S})$ and we take the scalar product with $\Delta_{q} U$ to obtain

$$
\begin{aligned}
\frac{d}{d t}\left\|\Delta_{q} U\right\|_{L^{2}}^{2} & \leq\left|\left\langle\Delta_{q}(A(U) \cdot \nabla U) \mid \Delta_{q} U\right\rangle\right| \\
& \leq C 2^{-q(d / 2+1)} c_{q}\|U\|_{B_{2,1}^{1+d / 2}}\|\nabla U\|_{L^{\infty}}\left\|\Delta_{q} U\right\|_{L^{2}}
\end{aligned}
$$

It follows that

$$
\begin{aligned}
& 2^{q(d / 2+1)}\left\|\Delta_{q} U(t)\right\|_{L^{2}} \\
& \leq 2^{q(d / 2+1)}\left\|\Delta_{q} U_{0}\right\|_{L^{2}}+C \int_{0}^{t} c_{q}(\tau)\|U(\tau)\|_{B_{2,1}^{1+d / 2}}\|\nabla U(\tau)\|_{L^{\infty}} d \tau .
\end{aligned}
$$

Summing on $q$ yields

$$
\|U(t)\|_{B_{2,1}^{1+d / 2}} \leq\left\|U_{0}\right\|_{B_{2,1}^{1+d / 2}}+C \int_{0}^{t}\|U(\tau)\|_{B_{2,1}^{1+d / 2}}\|\nabla U(\tau)\|_{L^{\infty}} d \tau
$$

Applying Gronwall's lemma we find

$$
\|U(t)\|_{B_{2,1}^{1+d / 2}} \leq\left\|U_{0}\right\|_{B_{2,1}^{1+d / 2}} \exp \left(C \int_{0}^{t}\|\nabla U(\tau)\|_{L^{\infty}}\right) d \tau
$$

Next we use that $B^{d / 2} \subset L^{\infty}$ to write

$$
\|\nabla U(t)\|_{L^{\infty}} \leq\|U(t)\|_{B_{2,1}^{1+d / 2}} \leq\left\|U_{0}\right\|_{B_{2,1}^{1+d / 2}} \exp \left(C \int_{0}^{t}\|\nabla U(\tau)\|_{L^{\infty}} d \tau\right) .
$$

If we note

$$
f(t)=C \int_{0}^{t}\|\nabla U(\tau)\|_{L^{\infty}} d \tau
$$

we obtain

$$
f^{\prime}(t) \leq C\left\|U_{0}\right\|_{B_{2,1}^{1+d / 2}} \exp (f(t)) .
$$

Again by Gronwall's lemma it follows

$$
\exp (-f(t)) \geq \exp (-f(0))-C t\left\|U_{0}\right\|_{B_{2,1}^{1+d / 2}}
$$


Hence, as long as

$$
C t\left\|U_{0}\right\|_{B_{2,1}^{1+d / 2}}<1
$$

we have

$$
\int_{0}^{t}\|\nabla U(\tau)\|_{L^{\infty}} d \tau<\infty
$$

Standard $L^{2}$ estimates and the inequality above imply uniqueness of solutions. This completes the proof.

Acknowledgements. This work was completed while the author was member of Université Paris 6 and of the Institute of Mathematics of the Romanian Academy. The author thanks both institutions for their support.

\section{References.}

[1] Bony, J.-M., Calcul symbolique et propagation des singularités pour les équations aux dérivées partielles non linéaires. Ann. Sci. École Norm. Sup. 14 (1981), 209-246.

[2] Cannone, M., Ondelettes, Paraproduits et Navier-Stokes. Diderot éditeur, Arts et Sciences, 1995.

[3] Chemin, J.-Y., Remarques sur l'existence globale pour le systeme de Navier-Stokes incompressible. SIAM J. Math. Anal. 23 (1992), 20-28.

[4] Chemin, J.-Y., Lerner, N., Flot de champs de vecteurs non-lipschitziens et équations de Navier-Stokes. Journal of Differential Equations 121 (1995), 314-328.

[5] Chemin, J.-Y., Fluides parfaits incompressibles. Astérisque 230, 1995.

[6] Constantin, P., Foias, C., Navier-Stokes Equations. The University of Chicago Press, 1988.

[7] Fujita, H., Kato, T., On the Navier-Stokes initial value problem I. Arch. Rational Mech. Anal. 16 (1964), 269-315.

[8] Kozono, H., Yamazaki, M., Semilinear heat equations and the NavierStokes equation with distributions in new function spaces as initial data. Comm. Partial Diff. Equations 19 (1994), 959-1014.

[9] Planchon, F., Global strong solutions in Sobolev or Lebesgue spaces to the incompressible Navier-Stokes equations in $\mathbb{R}^{3}$. Ann. Inst. $H$. Poincare, Analyse non linéaire 13 (1996), 319-336. 
[10] Sablé-Tougeron, M., Régularité microlocale pour des problèmes aux limites non linéaires. Ann. Inst. Fourier 36 (1986), 39-82.

[11] Temam, R., Navier-Stokes Equations. North-Holland, 1984.

[12] Triebel, H., Theory of function spaces. Birkhäuser Verlag, 1983.

Recibido: 5 de junio de 1.996

Revisado: 11 de agosto de 1.997

Dragoş Iftimie

IRMAR

Université de Rennes 1

Campus de Beaulieu

35042 Rennes Cedex, FRANCE iftimie@maths. univ-rennes 1 .fr 